AperTO - Archivio Istituzionale Open Access dell'Università di Torino

\title{
Comparing functional responses in predator-infected eco-epidemics models
}

\section{This is the author's manuscript}

Original Citation:

\section{Availability:}

This version is available http://hdl.handle.net/2318/145427

since

Published version:

DOI:10.1016/j.biosystems.2013.06.002

Terms of use:

Open Access

Anyone can freely access the full text of works made available as "Open Access". Works made available under a Creative Commons license can be used according to the terms and conditions of said license. Use of all other works requires consent of the right holder (author or publisher) if not exempted from copyright protection by the applicable law. 


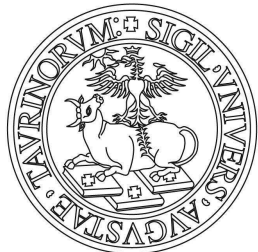 \\ UNIVERSITÀ DEGLI STUDI DI TORINO}

This is an author version of the contribution published on:

Mainul Haque, Sabiar Rahman, Ezio Venturino. Comparing functional responses in predator-infected eco-epidemics models. BioSystems, 114, 98-117, 2013.

The final version is available at:

http://www.journals.elsevier.com/biosystems/ 


\title{
Comparing functional responses in predator-infected eco-epidemics models
}

\author{
Mainul Haque ${ }^{a}$, Sabiar Rahman ${ }^{b}$, Ezio Venturino ${ }^{\dagger}$ \\ ${ }^{a}$ Division of Anaesthesia \& Intensive Care, \\ School of Clinical Sciences, Queen's Medical Centre, \\ University of Nottingham, UK. \\ ${ }^{b}$ Department of Mathematics, SSHS, WB, India. \\ † Dipartimento di Matematica, Giuseppe Peano, \\ Università di Torino, Torino, Italy \\ E-mails: mainul.haque@nottingham.ac.uk
}

\begin{abstract}
The current paper deals with the mathematical models of predator-prey system where a transmissible disease spreads among the predator species only. Four mathematical models are proposed and analysed with several popular predator functional responses in order to show the influence of functional response on eco-epidemic models. The existence, boundedness, uniqueness of solutions of all the models are established. Mathematical analysis including stability and bifurcation are observed. Comparison among the results of these models allows the general conclusion that relevant behaviour of the eco-epidemic predator-prey system, including switching of stability, extinction, persistence and oscillations for any species depends on four important parameters viz. the rate of infection, predator interspecies competition and the attack rate on susceptible predator. The paper ends with a discussion of the biological implications of the analytical and numerical results.
\end{abstract}

Keywords: Predator-Prey models; transmissible disease in predator; intra-specific competition among the predator; limit cycles; biological implications.

\section{Introduction}

In eco-epidemiology, (two) interacting populations are investigated, which also host a transmissible disease. The latter can affect only one population, the case most often treated in the literature, $[29,30,32]$, or it can spread to the other one(s). In fact, only a few fairly recent papers have dealt with this specific issue, $[19,33]$. The interactions can be either of competition type, [31], or else symbiotic, [16, 34], but mainly they are assumed of predatorprey nature, see the references in [21]. 
An important issue in models of this kind is the choice of the functional response. The latter is defined as the amount of prey consumed by one predator per unit of time. The functional response depends upon a number of factors. Some of them are represented by prey features, such as their ability to escape an attack, or the predators habits, e.g. the so-called prey handling time, the predators' search efficiency; finally others are represented by the natural environment, which establishes the structure of the prey habitat and the encounter rate among individuals of the different populations. These factors influence the predator's functional response, see [1, 2, 24], and therefore change the dynamical behaviour of the demographic model. Different responses have at times been considered in the context of eco-epidemiology, see for instance [15].

In this paper we consider a predator-prey demographic system, in which the predators are disease-affected so that the infected individuals are somewhat impaired in their hunting activities. Further, we assume that the epidemics cannot be transmitted to the prey. The main aim of this investigation is to uncover the effects of different functional responses in eco-epidemic models.

The most well-known predator-prey response is linear, called mass action or also Holling type I. It has the form $m x$, where $m$ is the predation rate and $x$ denotes the prey population. It is an adequate model if there is no handling time of the captured prey. This assumption is often regarded to be too crude, so as to adequately model the actual nature of the interactions, see [20]. In fact, predators often experience satiation so that their consumption rate declines with abundant available food, a property that is clearly not shown by a linear function. Despite this drawback, the mass action functional response is mathematically convenient, especially when the populations at hand are not too large. To better mimic the shape of the consumption rate, the Holling type II functional response $m x(a+x)^{-1}$ is used, [17]. It is a hyperbola with a horizontal asymptote, whose value reflects the maximum predation rate. Another variation that can be used is instead the Monod-Haldane or Holling type IV response function, mathematically represented by $m x\left(a^{2}+x^{2}\right)^{-1}$, [27]. It is a humped curve that raises up from the origin and then declines at high prey densities, this decline being attributed due to possible prey group defence. In fact a very well known epidemiological paper is based on such an assumption, but rather used for the disease transmission rate, [11], and more recent ones exploit the same feature, [9, 23]. Note that while Holling type II models predators' satiation, Holling type IV better describes situations in which the hunting drops when the prey density is sufficiently high, due for instance to prey toxicity.

The reasons for having different functional responses are various. They range from the fact that for instance a particular predator hunts prey having different escape abilities. If a particular prey has several natural predators, their different hunting techniques will induce diverse functional responses. The structure of the prey habitat may also alter their functional response. In addition also the prey behaviour is responsible for such changes. For instance the musk ox can more easily defend itself from wolves grouping in herds rather than staying alone. For other similar examples, see [12, 13].

The following more general Holling type IV functional response is sometimes used, see $[3,14,36]$,

$$
\frac{c x}{\frac{x^{2}}{b}+x+d} \text {. }
$$


Here the non-negative parameters $c$ and $d$ can be thought to be the maximum per capita consumption rate and the half-saturation constant in the absence of inhibitory effects. The parameter $b$ measures the predators immunity from the prey, since when it drops the predator's hunting efficiency decreases. Note indeed that for large $b$ the right hand side of (1.1) becomes a Holling type II functional response.

In particular in this paper we examine four eco-epidemic models differing in the type of response function, but to keep the length manageable one of them is seen only as a particular case. All of them contain the transmissible disease only among the predators. It affects the infected individuals by impairing somewhat their hunting efficiency.

Our aim is to make explicit and compare the dynamics of the proposed models and to identify the crucial system parameters that ensure specific population behaviours.

\section{Basic assumptions and mathematical model formula- tions}

Studies of predator-prey models have been performed in theoretical ecology since the early days of this discipline after the pioneering works of Lotka and Volterra. A number of predator-prey models have been proposed and studied during the last couple of decades (see [7], [8] and [5] and the references therein). A general predator-prey model (cf. [7] and [8]) in its classical form is represented by

$$
\frac{d x}{d t}=x \Gamma(x)-\bar{y} F(x), \quad \frac{d \bar{y}}{d t}=e F(x) \bar{y}-q \bar{y}
$$

where $x(t)$ and $\bar{y}(t)$ represent the densities of the prey and predator species, respectively, at time $t . F(x)$ denotes the prey per capita growth rate in the absence of predators and $q$ is the constant predator mortality. We call $F(x)$ the functional response and the term $e F(x) \bar{y}$ is known as the prey conversion factor into new predators. $e$ is the conversion efficiency constant. We assume that the prey population grows logistically with intrinsic growth rate $r$ and carrying capacity $\frac{r}{\beta}$ so that is $\Gamma(x)=r x\left(1-\frac{\beta x}{r}\right)$. A detailed history of modifications of the classical predator-prey model of the type (2.1) is given in reference [5]. This paper illustrates how, under the environmental stochasticity, the competition among the predator population is beneficial for a number of predator-prey models since it keeps them stable around their positive interior steady state (i.e. the situation in which both populations co-exist). Comparisons of the findings of this paper with the results of [4] allows the general conclusion that under both deterministic and stochastic environments, the predator-prey system benefits by the predator intra-species competition. This paper also resolves the ongoing 20-year controversy over the "paradox of enrichment" and illustrates how the model proposed in [4] would be the best possible predator-prey model among the most popular models discussed in the last half-century. The predator-prey model proposed in [4] contains an intra-specific competition term for the predator population. Therefore a more general predator-prey model would be 


$$
\frac{d x}{d t}=x \Gamma(x)-\bar{y} F(x), \quad \frac{d \bar{y}}{d t}=e F(x) \bar{y}-q y-h_{1} \bar{y}^{2}
$$

where $h_{1}$ represents the intra-specific competition of the predator population. The basic eco-epidemiological models are formulated from the general predator-prey model (2.2) under the following assumptions:

(A1): A transmissible unrecoverable disease spreads among the predators only. It can also be vertically transmitted. It does not affect the prey population. We assume that the disease spreads with an incidence rate $\lambda$ per unit time $t$. We split the predator population respectively among the sound predators $y(t)$ and the infected predators $z(t)$, so that $\bar{y}(t)=$ $y(t)+z(t)$.

(A2): The disease may reduce the hunting capabilities of the predator and thus we assume that the predation rate of the infected predators is reduced by a factor $0<p<1$.

(A3): We assume that the susceptible predators have a natural death rate $q$ and the infected predator has a higher death rate $\delta$ due to the disease related mortality, that is $\delta>q$. $\beta$ denotes the prey carrying capacity, $e<1$ the food conversion rate.

(A4): We also assume that the diseased predators are still able to compete with their similar for resources. Further we allow here for intra-specific competition, by writing the last term in both predators' equations; e.g. healthy predators $y$ feel the total predators population pressure at rate $h_{1}$ and similarly the infected ones $z$ feel it at rate $h_{2}$. All the parameters are assumed to be nonnegative.

With the above set of assumptions the model (2.2) reduces to

$$
\begin{aligned}
& \frac{d x}{d t}=r x-\beta x^{2}-F(x) y-p F(x) z \\
& \frac{d y}{d t}=e F(x) y-h_{1} y(y+z)-q y-\lambda y z \\
& \frac{d z}{d t}=\lambda y z+e p F(x) z-h_{2} z(y+z)-\delta z ;
\end{aligned}
$$

where $x(t)$ denotes the prey population, $y(t)$ the healthy predators and $z(t)$ the infected ones, at time $t$. The first equation describes the prey dynamics, accounting for logistic growth and hunting by the two types of predators. The second equation contains the sound predators' evolution, reproducing logistically in presence of food, and subject to natural mortality and to the disease contagion process. Diseased predators enter this class from the healthy class upon a "successful" contact with an infected one, first term in the third equation, reproduce logistically giving birth to diseased offspring in presence of food, and are subject to an additional mortality rate due to the disease.

Field Observation: [39] described a number of situations in which possible predators are infected by various transmissible diseases. Here we outline just the following pairs of parasites affecting hosts: rabies and foxes, Vulpes vulpes; Sarcoptes spp. affecting both 
foxes and coyotes, Canis latrans; Yersinia pestis and the Prairie dog, Cynomys spp.; Stomoxys calcitrans and Panthera leo; Aeromonas hydrophila and Alligator mississippiensis; we mention Phocine distemper virus affecting both the common seal, Phoca vitulina and the striped dolphin, Stenella coeruleoalba in the oceanic natural environment.

\subsection{Model I: linear functional response}

Let us take the functional response as the mass-action type, that is, $F(x)=m x$; then the general eco-epidemic model (2.3) reduces to

$$
\begin{aligned}
& \frac{d x}{d t}=r x-\beta x^{2}-m x y-p m x z, \\
& \frac{d y}{d t}=e m x y-h_{1} y(y+z)-q y-\lambda y z, \\
& \frac{d z}{d t}=\lambda y z+e m p x z-h_{2} z(y+z)-\delta z,
\end{aligned}
$$

The first equation describes the prey dynamics, accounting for logistic growth and hunting by the two types of predators. The second and third equations describe the predators dynamics. They contain mortality rates $q$ and $\delta$, which, in the absence of the prey, drive the predators to extinction. The second equation also contains the sound predators' evolution, reproducing logistically in presence of food, and subject to natural mortality and to the disease contagion process. Diseased predators enter this class from the healthy class upon a "successful" contact with an infected one, first term in the third equation, reproduce logistically giving birth to diseased offsprings in presence of food, and are subject to an additional mortality rate due to the disease. Note indeed that the diseased predators are still able to compete with their similar for resources.

The parameters, all always assumed to be non-negative, have the following interpretation: $r$ represents growth rate of the prey, $\frac{r}{\beta}$ the prey carrying capacity, $m$ the predation rate, $\lambda$ the contact rate, $e$ the conversion factor, $q$ the susceptible predator mortality, $h_{1}$ the intra and inter-specific competition rate for sound predators, $\delta>q$ the natural plus diseaserelated mortality of the infected predators, $h_{2}$ the intra and inter-specific competition rate of diseased predators. We assume that the hunting rate of the infected predators is reduced by a factor $p<1$ since the disease affects their hunting capabilities.

It is to be noted that the model (2.2) reduces to the famous predator-prey model of [37] if the functional response is taken to be mass-action type, that is, $F(x)=m x$. Therefore Model I represents an eco-epidemic model that is derived from the famous predator-prey model of Piolou [37] under the basic assumptions $A 1-A 4$. In view also of its resemblance to [32], we just basically summarize the analytic findings.

First of all, boundedness of the solution holds, (cf. Appendix 4.1). 


\subsubsection{The prey-only equilibrium}

The point $E_{1}^{I}\left(x_{I 1}, 0,0\right)$, with $x_{I 1}=r \beta^{-1}$ is stable if $P^{1 I} \equiv \frac{e r m}{\beta} \max \left\{\frac{p}{\delta}, \frac{1}{q}\right\}<1$. Thus $P^{1 I}>1$ makes the prey-only equilibrium unstable and is a threshold thus for the predators establishing themselves in the environment.

\subsubsection{The disease-free equilibrium}

$E_{3}^{I}\left(x_{I 3}, y_{I 3}, 0\right)$, with

$$
x_{I 3}=\frac{r h_{1}+q m}{e m^{2}+h_{1} \beta}, \quad y_{I 3}=\frac{r e m-q \beta}{e m^{2}+h_{1} \beta},
$$

and feasibility condition

$$
r e m>q \beta
$$

$E_{3}{ }^{I}$ is locally asymptotically stable if

$$
\frac{r-2 \beta x_{I 3}}{y_{I 3}}<m<m^{[I]} \equiv \min \left\{\frac{q+2 h_{1} y_{I 3}}{e x_{I 3}}, \frac{\delta+h_{2} y_{I 3}-\lambda y_{I 3}}{e p x_{I 3}}\right\} .
$$

In this case, by making the trace of the Jacobian at $E_{3}{ }^{I}$ vanish, Hopf bifurcations arise, when the bifurcation parameter $q$ crosses the value $q^{[I]}=2 \beta x_{I 3}+m y_{I 3}+2 h_{1} y_{I 3}-r-e m x_{I 3}$.

\subsubsection{The healthy predators-free equilibrium}

$E_{4}{ }^{I}\left(x_{I 2}, 0, z_{I 4}\right)$, where

$$
x_{I 4}=\frac{r h_{2}+p m \delta}{h_{2} \beta+p^{2} e m^{2}}, \quad z_{I 4}=\frac{e m p r-\delta \beta}{h_{2} \beta+p^{2} e m^{2}},
$$

is feasible for

$$
\text { empr }>\delta \beta
$$

and locally asymptotically stable if

$$
\delta<\delta^{[I]} \equiv \frac{-e m r h_{2}+\lambda e m p r+q h_{2} \beta+q p^{2} e m^{2}+h_{1} e m p r}{e m^{2} p+\lambda \beta+h_{1} \beta} .
$$




\subsubsection{The coexistence equilibrium}

$E_{5}^{I}\left(x_{I 5}, y_{I 5}, z_{I 5}\right)$ with

$$
\begin{aligned}
x_{I 5} & =\frac{r h_{1} \lambda-m \delta h_{1}+r \lambda^{2}+m p \lambda q-m p h_{2} q-m \lambda \delta+m h_{2} q+m p h_{1} \delta-r \lambda h_{2}}{h_{2} e m^{2}-p h_{2} e m^{2}-e m^{2} p h_{1}+p^{2} h_{1} e m^{2}-\beta \lambda h_{2}+\beta h_{1} \lambda+\beta \lambda^{2}}, \\
y_{I 5} & =\frac{e m r h_{2}-e m p r h_{1}-e m p r \lambda+\delta e m^{2} p-q e m^{2} p^{2}-q \beta h_{2}+\delta \beta h_{1}+\beta \lambda \delta}{h_{2} e m^{2}-p h_{2} e m^{2}-e m^{2} p h_{1}+p^{2} h_{1} e m^{2}-\beta \lambda h_{2}+\beta h_{1} \lambda+\beta \lambda^{2}}, \\
z_{I 5} & =\frac{e m p r h_{1}-\delta \beta h_{1}-e m r h_{2}-\delta e m^{2}+q e m^{2} p+\lambda e m r+q \beta h_{2}-\lambda q \beta}{h_{2} e m^{2}-p h_{2} e m^{2}-e m^{2} p h_{1}+p^{2} h_{1} e m^{2}-\beta \lambda h_{2}+\beta h_{1} \lambda+\beta \lambda^{2}} .
\end{aligned}
$$

and

$$
\begin{aligned}
& \frac{e m p r h_{1}+e m p r \lambda-e m r h_{2}+q e m^{2} p^{2}+q \beta h_{2}}{e m^{2} p+\beta h_{1}+\beta \lambda}<\delta<\delta^{[*]}, \\
& \frac{e m^{2}(1-p)\left(p h_{1}-h_{2}\right)}{\lambda^{2}-\lambda\left(h_{2}-h_{1}\right)}<\beta \\
& \lambda>h_{2}-h_{1} \\
& \delta^{[*]}=\min \left\{\frac{r h_{1} \lambda+r \lambda^{2}+m p \lambda q-m p h_{2} q+m h_{2} q-r \lambda h_{2}}{m h_{1}+m \lambda-m p h_{1}},\right. \\
& \left.\frac{e m p r h_{1}-e m r h_{2}+q e m^{2} p+\lambda e m r+q \beta h_{2}-\lambda q \beta}{\beta h_{1}+e m^{2}}\right\} .
\end{aligned}
$$

For this equilibrium we show local stability with the method of first approximation and also its global stability. We summarize the local and global stability results of model I through the following propositions whose proofs are given in Appendix A.

Proposition 1. The equilibrium $E_{5}{ }^{I}$ is locally asymptotically stable if

$$
\begin{array}{r}
\frac{r}{2 \beta}<x_{I 5}<\mu^{[I]}, \\
h_{2}<\lambda<h_{2}+m, \\
\delta>\left(\lambda-h_{2}\right) y_{I 5}+\left(p\left(\lambda+h_{1}\right)-2 h_{2}\right) z_{I 5}+e m p x_{I 5},
\end{array}
$$

where

$$
\begin{array}{r}
\mu^{[I]}=\min \left\{\frac{\lambda z_{I 5}+q+h_{1}\left(2 y_{I 5}+z_{I 5}\right)}{e m}, \frac{y_{I 5}\left(h_{2}-\lambda\right)+2 h_{2} z_{I 5}+\delta}{e m p},\right. \\
\left.\frac{y_{I 5}\left(2 h_{1}-\lambda-h_{2}\right)+z_{I 5}\left(\lambda+h_{1}-2 h_{2}\right)+q-\delta}{e m(1+p)}\right\} .
\end{array}
$$

Proposition 2. The coexistence equilibrium $E_{5}{ }^{I}$ is globally asymptotically stable if

$$
\frac{\lambda+3 h_{1}}{2\left(\lambda-h_{2}\right)} \leq \min \left\{\frac{\lambda+h_{1}+e m p}{2\left(\lambda-3 h_{2}+e m p\right)}, \frac{m p+2 \beta}{2 m p}\right\},
$$


Proof. Proof is given in Appendix 4.8.3.

\subsection{Model II: Holling type II functional response}

If we assume the functional response to be Holling type II, that is, $F(x)=\frac{m x}{a+x}$, then the classical model (2.2) reduces to the popular predator-prey model which has been proposed by Bazykin. A detailed study has been done by him and his colleagues, [38]. Using of the same functional response of model (2.3) gives a corresponding eco-epidemic model

$$
\begin{aligned}
\frac{d x}{d t} & =r x-\beta x^{2}-\frac{m x y}{a+x}-\frac{p m x z}{a+x} \\
\frac{d y}{d t} & =\frac{e m x y}{a+x}-\lambda y z-q y-h_{1} y(y+z), \\
\frac{d z}{d t} & =\lambda y z+\frac{e m p x z}{a+x}-\delta z-h_{2} z(y+z) .
\end{aligned}
$$

The parameters have the same meaning as for $(2.3)$; $a$ in this case represents the half saturation constant.

The Jacobian of the model (2.15) is given in Appendix B. From that we could easily see that the origin is an unstable equilibrium, in view of the Jacobian's eigenvalues $r,-\delta,-q$. Again $E_{2}{ }^{I I} \equiv E_{2}{ }^{I}$, since the system with no prey coincides with Model I, and therefore it must be infeasible for the same reason.

\subsubsection{The prey-only equilibrium}

The point $E_{1}^{I I}\left(x_{I I 1}, 0,0\right)$, with $x_{I I 1}=\frac{r}{\beta}$ is stable if the following condition holds

$$
\beta>\gamma^{[I I]} \equiv \max \left\{\frac{r(e m-q)}{a q}, \frac{r(e m p-\delta)}{a \delta}\right\},
$$

which can be recast in the form

$$
P^{1 I I} \equiv \frac{e r m}{r+a \beta} \max \left\{\frac{1}{q}, \frac{p}{\delta}\right\}<1 .
$$

\subsubsection{The disease-free equilibrium}

The point $E_{3}^{I I}\left(x_{I I 3}, y_{I I 3}, 0\right)$, with $y_{I I 3}=m^{-1}\left(r a+r x_{I I 3}-a \beta x_{I I 3}-\beta x_{I I 3}^{2}\right)$ and where $x_{I I 3}$ is a real positive root of the equation in $Z$

$$
h_{1} \beta Z^{3}+\left(2 h_{1} \beta a-h_{1} r\right) Z^{2}+\left(-2 h_{1} r a+h_{1} \beta a^{2}-q m+e m^{2}\right) Z-h_{1} r a^{2}-q a m=0 .
$$

is feasible for

$$
r>\beta x_{I I 3} .
$$


The characteristic equation of the Jacobian $J_{I I 3}=\left(\theta_{i j}\right), i, j=1,2,3$, factors to give one eigenvalue directly, and the two roots of the quadratic in $\tau$

$$
\tau^{2}-\left(\theta_{11}+\theta_{22}\right) \tau+\theta_{11} \theta_{22}-\theta_{21} \theta_{12}=0
$$

Assume now

$$
\frac{\left(r-2 \beta x_{3}\right)\left(a+x_{3}\right)^{2}}{a y_{3}}<m<m^{[I I]} \equiv \frac{a+x_{3}}{e x_{3}} \min \left\{q+2 h_{1} y_{3}, \frac{\delta+h_{2} y_{3}-\lambda y_{3}}{p}\right\} .
$$

Then

$$
\begin{array}{r}
\theta_{11}=r-2 \beta x_{I I 3}-\frac{m y_{I I 3}}{a+x_{I I 3}}+\frac{m x_{I I 3} y_{I I 3}}{\left(a+x_{I I 3}\right)^{2}}<0, \quad \theta_{12}=-\frac{m x_{I I 3}}{a+x_{I I 3}}<0 \\
\theta_{21}=\frac{e m y_{I I 3}}{a+x_{I I 3}}-\frac{e m x_{I I 3} y_{I I 3}}{\left(a+x_{I I 3}\right)^{2}}>0, \quad \theta_{22}=\frac{e m x_{I I 3}}{a+x_{I I 3}}-q-2 h_{1} y_{I I 3}<0 \\
\theta_{33}=\lambda y_{I I 3}+\frac{e m p x_{I I 3}}{a+x_{I I 3}}-\delta-h_{2} y_{I I 3}<0 .
\end{array}
$$

Both roots of the quadratic characteristic equation then have negative real parts. Therefore $E_{3}{ }^{I I}$ is locally asymptotically stable if (2.18) holds.

\subsubsection{The healthy predators-free equilibrium}

Here only prey $x$ and infected predators $z$ are present, $E_{4}{ }^{I I}\left(x_{I I 4}, 0, z_{I I 4}\right)$, with $z_{I I 4}=$ $(m p)^{-1}\left(r a+r x_{I I 4}-\beta a x_{I I 4}-\beta x_{I I 4}^{2}\right)$ and where $x_{I I 4}$ is a real positive root of the cubic

$$
h_{2} Z^{3} \beta+\left(2 h_{2} a \beta-r h_{2}\right) Z^{2}+\left(-2 h_{2} a r+h_{2} a^{2} \beta+p^{2} e m^{2}-p m \delta\right) Z-h_{2} a^{2} r-\delta a m p=0 .
$$

The equilibrium $E_{4}{ }^{I I}$ is feasible for

$$
r>x_{I I 4} \beta
$$

The entries of its Jacobian, $J_{I I 4}=\left(\psi_{i j}\right), i, j=1,2,3$ have the following signs

$$
\begin{aligned}
& \psi_{11}=r-2 \beta x_{I I_{4}}-\frac{p m z_{I I_{4}}}{a+x_{I I_{4}}}+\frac{p m x_{I I_{4}} z_{I I_{4}}}{\left(a+x_{I I_{4}}\right)^{2}}<0, \quad \psi_{13}=-\frac{p m x_{I I_{4}}}{a+x_{I I_{4}}}<0, \\
& \psi_{31}=\frac{e m p z_{I I_{4}}}{a+x_{I I_{4}}}-\frac{e m p x_{I I_{4}} z_{I I_{4}}}{\left(a+x_{I I_{4}}\right)^{2}}>0, \quad \psi_{33}=\frac{e m p x_{I I_{4}}}{a+x_{I I_{4}}}-\delta-2 h_{2} z_{I I_{4}}<0,
\end{aligned}
$$

if the following inequalities hold

$$
\begin{gathered}
\frac{\left(r-2 \beta x_{I I 4}\right)\left(a+x_{I I 4}\right)^{2}}{a p z_{I I 4}}<m<m^{[I I 1]} \\
\equiv \min \left\{\frac{\left(q+h_{1} z_{I I 4}+\lambda z_{I I 4}\right)\left(a+x_{I I 4}\right)}{e x_{I I 4}}, \frac{\left(\delta+2 h_{2} z_{I I 4}\right)\left(a+x_{I I 4}\right)}{e p x_{I I 4}}\right\} .
\end{gathered}
$$

Hence, since the characteristic equation factors once more to give one eigenvalue and the following quadratic in $\tau$ having roots with negative real parts,

$$
\tau^{2}-\left(\psi_{11}+\psi_{33}\right) \tau+\left(\psi_{11} \psi_{33}-\psi_{13} \psi_{31}\right)=0
$$


stability holds if (2.20) is satisfied.

\subsubsection{The coexistence equilibrium}

The equilibrium $E_{5}^{I I}\left(x_{I I 5}, y_{I I 5}, z_{I I 5}\right)$ has analytically determined components

$$
\begin{aligned}
y_{I I 5} & =-\frac{x_{I I 5}\left(h_{1} p e m+e m p \lambda-\lambda \delta+h_{2} q-h_{2} e m-\delta h_{1}\right)-a \lambda \delta-a \delta h_{1}+a h_{2} q}{\lambda\left(\lambda+h_{1}-h_{2}\right)\left(a+x_{I I 5}\right)}, \\
z_{I I 5} & =\frac{x_{I I 5}\left(h_{1} p e m-\delta h_{1}+h_{2} q-h_{2} e m-\lambda q+\lambda e m\right)-a h_{1} \delta+a h_{2} q-a q \lambda}{\lambda\left(\lambda+h_{1}-h_{2}\right)\left(a+x_{I I 5}\right)} .
\end{aligned}
$$

where $x_{I I 5}$ is a real positive root of the cubic equation

$$
\begin{aligned}
& \left(-h_{2} \beta \lambda+\lambda \beta h_{1}+\lambda^{2} \beta\right) Z^{3}+\left(2 \lambda a \beta h_{1}+h_{2} r \lambda-\lambda r h_{1}-2 h_{2} a \beta \lambda-\lambda^{2} r+2 \lambda^{2} a \beta\right) Z^{2} \\
& +\left(-\lambda p q m-h_{2} p e m^{2}+\delta m \lambda+\delta m h_{1}+h_{2} e m^{2}-h_{2} q m-2 \lambda^{2} a r+\lambda^{2} a^{2} \beta-h_{2} a^{2} \beta \lambda\right. \\
& \left.+e m^{2} p^{2} h_{1}-e m^{2} p h_{1}-\delta m h_{1} p-2 \lambda a r h_{1}+\lambda a^{2} \beta h_{1}+h_{2} p q m+2 h_{2} a r \lambda\right) Z-\lambda p q a m \\
& -h_{2} q a m-\lambda a^{2} r h_{1}+\delta a m \lambda+\delta a m h_{1}+h_{2} a^{2} r \lambda-\lambda^{2} a^{2} r+h_{2} p q a m-\delta a m h_{1} p=0 .
\end{aligned}
$$

$E_{5}{ }^{I I}$ is feasible for

$$
\begin{aligned}
\delta<\delta^{[I I]} \equiv & \min \left\{\frac{x_{I I 5}\left(h_{1} p e m-\lambda q-h_{2} e m+e m \lambda+h_{2} q\right)+a h_{2} q-a q \lambda}{h_{1}\left(a+x_{I I 5}\right)},\right. \\
& \left.\frac{x_{I I 5}\left(\lambda e m p+q h_{2}+h_{1} p e m-h_{2} e m\right)+a h_{2} q}{\left(h_{1}+\lambda\right)\left(a+x_{I I 5}\right)}\right\}, \quad \lambda>h_{2}-h_{1} .
\end{aligned}
$$

We summarize the behaviour of the model around $E_{5}{ }^{I I}$ through the following propositions whose proofs are given in Appendix C.

Proposition 3. Let

$$
\lambda^{[I I]}=\min \left\{\frac{h_{2}}{y_{I I 5}}\left(y_{I I 5}+p z_{I I 5}\right), \frac{h_{2}-p h_{1}}{p}\right\}, \quad \mu^{[I I]}=\frac{m\left(y_{I I 5}+p z_{I I 5}\right)}{\left(a+x_{I I 5}\right)^{2}} .
$$

The system $(2.15)$ is locally asymptotically stable at $E_{5}{ }^{I I}$ if the following conditions hold

$$
a>x_{I I 5}, h_{2}<\lambda<\lambda^{[I I]}, \mu^{[I I]}<\beta<\mu^{[I I]}+h_{2} \frac{z_{I I 5}}{x_{I I 5}}, \lambda^{[I I]}<\lambda<\mu^{[I I]}, \frac{r}{2 \beta}<x_{I I 5} .
$$

Proposition 4. The coexistence equilibrium $E_{5}^{I I}$ is globally asymptotically stable if, 
recalling the boundedness property of the prey populations,

$$
\begin{array}{r}
\beta>m \frac{p z_{I I 5}+y_{I I 5}}{a\left(a+x_{I I 5}\right)}, \\
4 h_{1}\left[\beta-m \frac{\left.p z_{I I 5}+y_{I I 5}\right]}{a\left(a+x_{I I 5}\right)}>\left(\frac{m\left(a(1-e)+x_{I I 5}\right)}{a\left(a+x_{I I 5}\right)}\right)^{2},\right. \\
{\left[\frac{p m(1-e)}{a+W}+\frac{e m p x_{I I 5}}{\left(a+x_{I I 5}\right)(a+W)}\right]\left[\frac{(1-e) m}{a+W}+\frac{e m p x_{I I 5}}{(a+W)\left(a+x_{I I 5}\right)}\right]} \\
>4 \frac{\left(h_{1}+h_{2}\right)}{2}\left[\beta-\frac{p m z_{I I 5}+m y_{I I 5}}{a\left(a+x_{I I 5}\right)}\right], \\
h_{1}+h_{2}>2 h_{1} p .
\end{array}
$$

\subsection{Models III and IV: Holling types III and IV functional re- sponses}

Once we use the functional response $F(x)=\frac{m x}{a^{2}+b x+x^{2}}$ in the general eco-epidemic model (2.3), it reduces to following model which we call Model IV.

$$
\begin{aligned}
& \frac{d x}{d t}=r x-\beta x^{2}-\frac{m x y}{a^{2}+b x+x^{2}}-\frac{p m x z}{a^{2}+b x+x^{2}}, \\
& \frac{d y}{d t}=\frac{e m x y}{a^{2}+b x+x^{2}}-\lambda y z-q y-h_{1} y(y+z), \\
& \frac{d z}{d t}=\lambda y z+\frac{e m p x z}{a^{2}+b x+x^{2}}-\delta z-h_{2} z(y+z),
\end{aligned}
$$

The particular case $b=0$ is not treated explicitly, but corresponds to what we call Model III. The Jacobian of the model (2.27) is given in Appendix D. A straightforward calculation form the Jacobian matrix shows that the origin with eigenvalues $r,-\delta,-q$ is unstable. Once more, $E_{2}{ }^{I V} \equiv E_{2}{ }^{I}$ and infeasibility follows.

\subsubsection{The prey-only equilibrium}

At $E_{1}^{I V}\left(x_{I V 1}, 0,0\right)$, with $x_{I V 1}=r \beta^{-1}$ stability is ensured by

$$
a^{2} \beta^{2}+r^{2}+b r \beta>\operatorname{emr} \beta \max \left\{\frac{1}{q}, \frac{p}{\delta}\right\} .
$$




\subsubsection{The disease-free equilibrium}

$E_{3}{ }^{I V}\left(x_{I V 3}, y_{I V 3}, 0\right)$, with

$$
y_{I V 3}=\frac{1}{m}\left[r x_{I V 3}^{2}+r b x_{I V 3}+r a^{2}-\beta x_{I V 3}^{3}-\beta x_{I V 3}^{2} b-\beta x_{I V 3} a^{2}\right]
$$

and where $x_{I V 3}$ is a real positive root of the quintic equation

$$
\begin{array}{r}
h_{1} Z^{5} \beta+\left(2 h_{1} \beta b-h_{1} r\right) Z^{4}+\left(2 h_{1} \beta a^{2}+h_{1} b^{2} \beta-2 h_{1} r b\right) Z^{3} \\
+\left(2 h_{1} b \beta a^{2}-h_{1} b^{2} r-2 h_{1} r a^{2}-q m\right) Z^{2} \\
+\left(h_{1} a^{4} \beta-q b m-2 h_{1} b r a^{2}+e m^{2}\right) Z-q a^{2} m-h_{1} a^{4} r=0,
\end{array}
$$

is feasible if

$$
r>\beta x_{I V 3} .
$$

The Jacobian matrix at $E_{3}{ }^{I V}$ is denoted by $J_{I V 3}=\left(\omega_{i j}\right), i, j=1,2,3$ with the relevant entries given in Appendix 4.6. The characteristic equation also factors, giving an eigenvalue

$$
\lambda y_{I V 3}+\frac{e m p x_{I V 3}}{a^{2}+b x_{I V 3}+x_{I V 3}{ }^{2}}-\delta-h_{2} y_{I V 3}
$$

and a quadratic characteristic equation in $\tau$

$$
\tau^{2}-\left(\omega_{11}+\omega_{22}\right) \tau+\omega_{11} \omega_{22}-\omega_{21} \omega_{12}=0
$$

whose roots have negative real parts since $\omega_{11}<0, \omega_{22}<0, \omega_{21}>0, \omega_{12}<0$, assuming

$$
\begin{array}{r}
a>x_{I V 3}, \quad \frac{\left(r-2 \beta x_{I V 3}\right)\left(a^{2}+b x_{I V 3}+x_{I V 3}^{2}\right)^{2}}{\left(a^{2}-x_{I V 3}{ }^{2}\right) y_{I V 3}} \equiv m_{[I V]}<m<m^{[I V]} \\
\equiv \frac{a^{2}+b x_{I V 3}+x_{I V 3}{ }^{2}}{e x_{I V 3}} \min \left\{q+2 h_{1} y_{I V 3}, \frac{\delta+h_{2} y_{I V 3}-\lambda y_{I V 3}}{p}\right\} .
\end{array}
$$

Conditions (2.30) thus ensure stability.

\subsubsection{The healthy predator-free equilibrium}

We now consider the point $E_{4}{ }^{I V}\left(x_{I V 4}, 0, z_{I V 4}\right)$, with

$$
z_{I V 4}=\frac{1}{p m}\left[x_{I V 4}^{2}+b x_{I V 4}+a^{2}\right]\left(r-x_{I V 4} \beta\right) .
$$

Here $x_{I V 4}$ is any real positive root of the equation

$$
\begin{array}{r}
h_{2} Z^{5} \beta+\left(2 h_{2} \beta b-h_{2} r\right) Z^{4}+\left(2 h_{2} \beta a^{2}+h_{2} b^{2} \beta-2 h_{2} r b\right) Z^{3} \\
+\left(2 h_{2} b \beta a^{2}-h_{2} b^{2} r-2 h_{2} r a^{2}-\delta m p\right) Z^{2} \\
+\left(-2 h_{2} b r a^{2}+e m^{2} p^{2}+h_{2} a^{4} \beta-\delta b m p\right) Z-\delta a^{2} m p-h_{2} a^{4} r=0
\end{array}
$$


Note that by Descartes' rule, at least one such root exists. $E_{4}{ }^{I V}$ is feasible if $z_{I V 4} \geq 0$ i.e. for

$$
r>x_{I V 4} \beta \text {. }
$$

The characteristic equation stemming from the Jacobian at $E_{4}{ }^{I V}$, denoted by $J_{I V 4}=\left(v_{i j}\right)$, $i, j=1,2,3$ factors to give the eigenvalue

$$
v_{22}=\frac{e m x_{I V_{4}}}{x_{I V_{4}}^{2}+b x_{I V_{4}}+a^{2}}-\lambda z_{I V_{4}}-q-h_{1} z_{I V_{4}}
$$

and the roots of the quadratic $\tau^{2}-\left(v_{11}+v_{33}\right) \tau+v_{11} v_{33}-v_{13} v_{31}=0$. Take

$$
\begin{aligned}
a & >x_{I V 4}, \quad \frac{\left(r-2 \beta x_{I V 4}\right)\left(a^{2}+b x_{I V 4}+x_{I V 4}^{2}\right)^{2}}{\left(a^{2}-x_{I V 4}^{2}\right) p z_{I V 4}} \equiv m_{\left[I V_{1}\right]}<m<m^{\left[I V_{1}\right]} \\
& \equiv \frac{a^{2}+b x_{I V 4}+x_{I V 4}^{2}}{e x_{I V 4}} \min \left\{\left(q+h_{1} z_{I V 4}+\lambda z_{I V 4}\right), p^{-1}\left(\delta+2 h_{2} z_{I V 4}\right)\right\}
\end{aligned}
$$

to find that $v_{11}<0, v_{13}<0, v_{22}<0, v_{31}>0$ and $v_{33}<0$, conditions that by the Routh-Hurwitz criterion thus ensure stability.

\subsubsection{The coexistence equilibrium}

In spite of the complexity of the system, the coexistence equilibrium can be analytically determined. In view of this fact, although the expressions are involved we decided to report them below. The population levels at $E_{5}{ }^{I V}\left(x_{I V 5}, y_{I V 5}, z_{I V 5}\right)$ are then

$$
\begin{aligned}
y_{I V 5} & =\frac{b_{1}}{m\left(a^{2}+x_{I V 5}^{2}+b x_{I V 5}\right) \lambda\left(\lambda+h_{1}-h_{2}\right)}, \\
z_{I V 5} & =\frac{d_{1}}{\left(a^{2}+x_{I V 5} b+x_{I V 5}^{2}\right) \lambda\left(\lambda+h_{1}-h_{2}\right)\left(h_{1} p-\lambda-h_{1}\right)},
\end{aligned}
$$

where the numerators $b_{1}$ has a complicated expressions and given in Appendix 4.8.4.

Furthermore, $x_{I V 5}$ is a real positive root of the equation

$$
\sum_{i=0}^{5} A_{i} I^{i}=0
$$

with coefficients given in Appendix 4.8.1. In general, this equation has five roots. The sufficient conditions for which the equation (2.33) has a positive root are presented in Appendix 4.8.2.

We summarize the behaviour of the system (2.27) around the equilibrium point $E_{5}{ }^{I V}\left(x_{I V 5}, y_{I V 5}, z_{I V 5}\right)$ by the following propositions whose Proofs are given in Appendix . 
Proposition 5. The equilibrium $E_{5}{ }^{I V}$ is locally asymptotically stable if

$$
\begin{array}{r}
a>x_{I V 5}, \quad h_{2}<\lambda<\lambda^{[I V]}, \quad \mu^{[I V]}<\beta<\mu^{[I V]}+h_{2} \frac{z_{I V 5}}{x_{I V 5}}, \\
\lambda^{[I V]}=\min \left\{\frac{h_{2}}{y_{I V 5}}\left(y_{I V 5}+p z_{I V 5}\right), p^{-1}\left(h_{2}-p h_{1}\right)\right\}, \\
\mu^{[I V]}=\frac{m\left(2 x_{I V 5}+b\right)\left(y_{I V 5}+p z_{I V 5}\right)}{\left(a^{2}+b x_{I V 5}+x_{I V 5}\right)^{2}} .
\end{array}
$$

Proposition 6. The coexistence equilibrium $E_{5}{ }^{I V}$ is globally asymptotically stable if, recalling the boundedness property of the prey population,

$$
\begin{array}{r}
\beta>m \frac{\left(p z_{I V 5}+y_{I V 5}\right)\left(b+W+x_{I V 5}\right)}{a^{2}\left(a^{2}+b x_{I V 5}+x_{I V 5}^{2}\right)}, \quad h_{1}+h_{2}>2 h_{1} p, \\
4 h_{1}\left[\beta-m \frac{\left(p z_{I V 5}+y_{I V 5}\right)\left(b+W+x_{I V 5}\right)}{a^{2}\left(a^{2}+b x_{I V 5}+x_{I V 5}^{2}\right)}\right] \\
>m^{2}\left(\frac{(1-e)\left(a^{2}+x_{I V 5}^{2}\right)+b x_{I V 5}+e m x_{I V 5}\left(W+x_{I V 5}\right)}{a^{2}\left(a^{2}+b x_{I V 5}+x_{I V 5}^{2}\right)}\right)^{2}, \\
\frac{p m^{2}}{4}\left(\frac{1-e}{a^{2}+b W+W^{2}}+\frac{e x_{I V 5}\left(b+x_{I V 5}\right)}{\left.a^{2}+b W+W^{2}\right)\left(a^{2}+b x_{I V 5}+x_{I V 5}^{2}\right)}\right)^{2} \\
>\frac{\beta\left(h_{1}+h_{2}\right)}{2}-\frac{m\left(b+x_{I V 5}\right)\left(y_{I V 5}+p z_{I V 5}\right)\left(h_{1}+h_{2}\right)}{2\left(a^{2}+b W+W^{2}\right)\left(a^{2}+b x_{I V 5}+x_{I V 5}^{2}\right)} .
\end{array}
$$

Proposition 7. The system possesses a Hopf bifurcation around $E_{5}{ }^{I V}$ when $m$ passes through $m_{c r}$, where $m_{c r}$ satisfy the equality $K_{1} K_{2}=K_{3}$ and $K_{i}$ 's satisfy the characteristic equation of the system $(2.27)$ at $E_{5}^{I V}\left(x_{I V 5}, y_{I V 5}, z_{I V 5}\right)$, that is,

$$
\hat{\omega}^{3}+K_{1} \hat{\omega}^{2}+K_{2} \hat{\omega}+K_{3}=0
$$

Proof. The proof is given in Appendix 4.6.

\section{Numerical simulations and discussion.}

For the convenience of the reader, we report all the conditions of local and global stability for the various equilibria in Tables $1-3$, including also Model III, which as mentioned is the particular case for $b=0$ of Model IV.

In all models, the trivial equilibrium is always unstable for all possible parameter values. This is a good result, since it means that the ecosystem will never disappear.

The prey-only equilibrium in Models I and II is stable if $P^{1 I}$ and $P^{1 I I}$ are smaller than 1. Note that Model II is favoured for stability, in the sense that the half saturation constant 
helps in lowering the critical ratio $P^{1 I I}$. Thus, when predators experience satiation, modelled by the Holling type II term, it is harder for them to invade the ecosystem. Prey in such case thrives at the system's carrying capacity. Other factors that favour the stability of this equilibrium in both models in the same way, are a low predation rate $m$, low conversion factor $e$, low hunting rate of infected predators, expressed by the parameter $p$. Equivalently, we need large healthy $q$ and infected $\delta$ predators' mortalities. The role of the prey reproduction rate $r$ instead is not clear-cut in Model II, while we need it low in Model I. This is quite unexpected, but it can be explained by the fact that if prey reproduce fast, they favour the predators growth and thus ultimately the possibility of their establishing themselves in the ecosystem. It is hard to compare the first pair of Models with the second one. But for the second pair, it is clear that for Model IV stability is favoured, since a positive value of $b$ will help in satisfying it, even if the same condition does not hold for Model III.

The prey-free equilibrium is infeasible in all the models. This makes sense since in absence of prey, the predators have no source of food, and hence the latter must get extinguished, first the healthy ones and then the infected ones.

From the feasibility conditions and the stability analysis of the equilibria, we observe that the parameters $\lambda, m, h_{1}$ and $h_{2}$ play a crucial role in controlling the dynamical behaviour of the models. In all the four models the trivial equilibrium is always an unstable saddle for all parameter values.

Now $E_{1}{ }^{n}$ is locally asymptotically stable if $\left(P^{1 I}\right)<1, P^{1 I I}<1,\left\{a^{2} \beta^{2}+r^{2}>\operatorname{emr} \beta \max \left\{\frac{1}{q}, \frac{p}{\delta}\right\}\right\}$ and $\left\{a^{2} \beta^{2}+r^{2}+b r \beta>\operatorname{emr} \beta \max \left\{\frac{1}{q}, \frac{p}{\delta}\right\}\right\}$ for $n=\mathrm{I}$, II, III, IV. Biologically, it implies that if both the predation rate and the reduced factor of prey be low, then the healthy predator and infected predator cannot survive and the system will be equilibrium only where prey exists. From the fifth row of Table 1 the stability conditions of $E_{3}{ }^{n}$ are $\left[\frac{r-2 \beta x_{I 3}}{y_{I 3}}\right.$ $\left.<m<m^{[I]} \equiv \min \left\{\frac{q+2 h_{1} y_{I 3}}{e x_{I 3}}, \frac{\delta+h_{2} y_{I 3}-\lambda y_{I 3}}{e p x_{I 3}}\right\}\right],\left[\frac{\left(r-2 \beta x_{I I 3}\right)\left(a+x_{I I 3}\right)^{2}}{a y_{I I 3}}\right.$ $\left.<m<m^{[I I]} \equiv \frac{a+x_{I I 3}}{e x_{I I 3}} \min \left\{q+2 h_{1} y_{I I 3}, \frac{\delta+h_{2} y_{I I 3}-\lambda y_{I I 3}}{p}\right\}\right],\left[(i) \frac{\left(r-2 \beta x_{I I I 3}\right)\left(a^{2}+x_{I I I 3}\right)^{2}}{\left(a^{2}-x_{I I I 3}\right) y_{I I I 3}} \equiv m_{[I I I]}<\right.$ $m<m^{[I I I]} \equiv \frac{a^{2}+x_{I I I 3}{ }^{2}}{e x_{I I I 3}}$ $\min \left\{q+2 h_{1} y_{I I I 3}, \frac{\delta+h_{2} y_{I I I 3}-\lambda y_{I I I 3}}{p}\right\}$,

(ii) $\left.a>x_{I I I 3}\right]$ and $\left[(i) \frac{\left(r-2 \beta x_{I V 3}\right)\left(a^{2}+b x_{I V}+x_{I V 3}\right)^{2}}{\left(a^{2}-x_{I V 3}\right) y_{I V 3}} \equiv m_{[I V]}<m<m^{[I V]} \equiv \frac{a^{2}+b x_{I V 3}+x_{I V 3}{ }^{2}}{e x_{I V 3}}\right.$ $\min \left\{q+2 h_{1} y_{I V 3}, \frac{\delta+h_{2} y_{I V 3}-\lambda y_{I V 3}}{p}\right\}$, (ii) $\left.a>x_{I V 3}\right]$, respectively which depend mainly predation rate $m$ which also depends on the reduced factor $p$ and $\lambda$. It is observed that for the lower value of $p$ and $\lambda$ the prey and healthy predator co-exist in the form of a stable equilibrium. But for the models II and III depend upon the half saturation constant $a$. For the model IV the co-existence (of prey and healthy predator) depends also the another ecological parameter $b$. Next from the sixth row of the Table 1 with used notations $\delta^{[I]} \equiv \frac{-e m r h_{2}+\lambda e m p r+q h_{2} \beta+q p^{2} e m^{2}+h_{1} e m p r}{e m^{2} p+\lambda \beta+h_{1} \beta}$, 
$m^{[I I 1]} \equiv \min \left\{\frac{\left(q+h_{1} z_{I I 4}+\lambda z_{I I 4}\right)\left(a+x_{I I 4}\right)}{e x_{I I 4}}, \frac{\left(\delta+2 h_{2} z_{I I 4}\right)\left(a+x_{I I 4}\right)}{e p x_{I I 4}}\right\}, \frac{\left(r-2 \beta x_{I I I 4}\right)\left(a^{2}+x_{I I I 4}{ }^{2}\right)^{2}}{\left(a^{2}-x_{I I I 4}\right) p z_{I I I 4}} \equiv m_{\left[I I I_{1}\right]}$,

$m^{\left[I I I_{1}\right]} \equiv \frac{a^{2}+x_{I I I 4}{ }^{2}}{e x_{I I I 4}} \min \left\{\left(q+h_{1} z_{I I I 4}+\lambda z_{I I I 4}\right), p^{-1}\left(\delta+2 h_{2} z_{I I I 4}\right)\right\}$

$\frac{\left(r-2 \beta x_{I V 4}\right)\left(a^{2}+b x_{I V 4}+x_{I V 4}\right)^{2}}{\left(a^{2}-x_{I V 4^{2}}\right) p z_{I V 4}} \equiv m_{\left[I V_{1}\right]}, m^{\left[I V_{1}\right]}$

$\equiv \frac{a^{2}+b x_{I V 4}+x_{I V 4}^{2}}{e x_{I V 4}} \min \left\{\left(q+h_{1} z_{I V 4}+\lambda z_{I V 4}\right), p^{-1}\left(\delta+2 h_{2} z_{I V 4}\right)\right\}$ one can observe from the stability conditions of $E_{4}{ }^{n}$ if the infection rate is too high and the predation rate moderate and the intra and inter competition rate $h_{2}$ very low then the healthy predator cannot survive and the system converges to the equilibrium where prey and infected predator co-exist.

Successful observation of local stability of $E_{5}{ }^{n}$ in the Table 1 allows the comparison of stability for the four models I II III and IV. We can then compare the situation for global stability of coexistence equilibrium for the different models.

To describe the coexistence of global stability for different models for comparison purposes we set the fixed parameter values specified in Table 4 and vary only the hunting rate on prey by healthy predator, $m$, and intra- and inter-specific competition rates $h_{1}, h_{2}$ of healthy predator and infected predator, respectively. For the above set of values of the parameters the coexistence equilibrium $E_{5}$ will be globally asymptotically stable if we choose the value of $m$ as 1, 1.002, 1.5 and 1.7 for the model I, II, III, IV respectively. At the same time the choosing set of values of $h_{1}$ and $h_{2}$ are $\{0.005,0.0001,0.00015,0.00017\}$ and $\{0.04,0.2$, $0.3,0.4\}$. In this case we observe that all the trajectories originating from any point of the basin of attraction converges to the equilibrium points $(1.85,5.99,1.01),(1.81,3.58,0.49)$ and $(1.86,10.90,0.34)$ up to two decimal places respectively for the models I, II, III, IV. which means that the system is globally asymptotically stable for these models.

The comparison of global stability for the models I and II is relatively simple. It is worth to compare the behaviours of models III and IV since both contain non monotonic response functions (cf., [25], [26]). It is analytically very complicated to study the problem in the parametrer space. But with numerical simulations, the parameter space $m-h_{1}-h_{2}$ of model IV has larger regions of global stability compared to the other three models. Similarly the region of global stability of model III is larger than the one of model II. The range of parameter values of the global stability region of model I is larger than the that of model II. This is not surprising. Several researchers used Holling type IV non monotonic response function motivating it as a group defence mechanism. Predation indeed decreases or is even prevented when the prey are many, so that they can better defend themselves, (cf. [13], [22], [35]). Our results indicate thus that in this way the interactions of predators and prey are stabilized for a wide range of parameter values.

Also we compare the bifurcation behaviour for our four models in Figure 2 choosing the same initial conditions. A general proof showing the existence of critical Hopf bifurcations for all models is given in the Appendix 4.6. We also obtained phase portraits of global stable behaviour of the four models but they are not reported here due to lack of space.

The model (2.27) is more general, we present the Hopf bifurcation analysis for this model only in Proposition 7.

For numerical simulation we take $m=5.5$, keeping the other parameter at the same value as that of Fig. 2(iv). All the feasibility conditions of the interior equilibrium of the system (2.27) hold; the co-existence equilibrium point is $E_{5}^{I V}(1.62,1.90,0.79)$. We obtain here 


\begin{tabular}{|c|c|}
\hline $\begin{array}{l}\text { Interior } \\
\text { Equilibria }\end{array}$ & Conditions For Global Stability \\
\hline$E_{5}{ }^{I V}$ & $\begin{array}{l}(i) b>a, \\
(i i) \beta>m \frac{\left(p z_{I V 5}+y_{I V 5}\right)\left(b+x_{I V 5}\right)}{a^{2}\left(a^{2}+b x_{I V 5}+x_{I V 5}^{2}\right)}, \\
(i i i) 4 h_{1}\left(\beta-m \frac{\left(p z_{I V 5}+y_{I V 5}\right)\left(b+W+x_{I V 5}\right)}{a^{2}\left(a^{2}+b x_{I V 5}+x_{I V 5}^{2}\right)}\right) \\
>\left(\frac{m\left(a(1-e)\left(a^{2}+x_{I V 5}^{2}\right)+e m x_{I V 5}\left(W+x_{I V 5}\right)\right.}{\left.a^{2}\left(a^{2}+b x_{I V 5}+x_{I V 5}^{2}\right)\right)}\right)^{2}, \\
(i v) \frac{p m^{2}}{4}\left(\frac{(1-e)}{a^{2}+b W+W^{2}}+\frac{e x_{I V 5}\left(b+x_{I V 5}\right)}{\left(a^{2}+b W+W^{2}\right)\left(a^{2}+b x_{I V 5}+x_{I V 5}^{2}\right)}\right)^{2} \\
>\frac{\beta\left(h_{1}+h_{2}\right)}{2}-\frac{m\left(b+x_{I V 5}\right)\left(y_{I V 5}+p z_{I V 5}\right)\left(h_{1}+h_{2}\right)}{2\left(a^{2}+b W+W^{2}\right)\left(a^{2}+b x_{I V 5} I V 5+x_{I V 5}^{2}\right)}, \\
\text { and }(v) h_{1}+h_{2}>2 h_{1} p .\end{array}$ \\
\hline
\end{tabular}

Table 1: Global stability conditions of the interior equilibrium $E_{5}{ }^{I V}$.

$m_{c r}=5.9$ and $m_{\max }=6.3$. The interior equilibrium point $E_{5}^{I V}$ is asymptotically stable when $m=5.5<m_{c r}$. This situation is presented in (a) of Fig. 1in terms of solution curves. Again when $m$ lies between $m_{c r}$ and the maximum value of $m_{c r}$ then a stable bifurcating limit cycle occurs as supercritical Hopf bifurcation (cf. Fig. 1 (b)). An extensive numerical simulation shows that when the value of $m$ is very close to $m_{c r}$, the three populations (prey, healthy predator and infected predator) take long time to become stable whereas when $m$ crosses the value $m_{c r}$ and becomes very close to $m_{\max }$, the three populations become unstable. Therefore we arrive at the following conclusion:

Proposition 8. In the interval $\left[0, m_{\max }\right]$, there exists a critical value $m_{c r}$, where a supercritical Hopf bifurcation occurs. For supercritical Hopf bifurcation in the interval $\left[0, m_{\max }\right]$ the interior equilibrium point is asymptotically stable between 0 and $m_{c r}$ and for $m$ lying between $m_{c r}$ and $m_{\max }$, a limit cycle occurs. On the other hand when $m>m_{\max }$, the equilibrium does not exist since the predation rate is too high.

An example of population dynamics for the Holling type IV response function phenomenon is described by Tener [28]. Lone musk Ox can be successfully attacked by wolves. small herds of musk Ox (2 to 6 animals) are attacked but with rare success. No successful attacks have been shown in larger herds. Another example observed by Holms and Bethel [18] involves certain insect individuals. Large swarms of insects make the individual identification difficult for their predators. 


\begin{tabular}{|c|c|c|c|c|}
\hline $\begin{array}{l}\text { Functional } \\
\text { response } \\
\text { Equilibria }\end{array}$ & Model $\mathbf{I}(\mathbf{n}=\mathbf{I})$ & Model II $(n=I I)$ & Model III $(n=I I I)$ & Model IV $(n=I V)$ \\
\hline$E_{0}^{n}$ & Always unstable & Always unstable & Always unstable & Always unstable \\
\hline$E_{1}{ }^{n}$ & $P^{11}<1$ & $P^{111}<1$ & $\begin{array}{l}a^{2} \beta^{2}+r^{2}> \\
\operatorname{emr} \beta \max \left\{\frac{1}{q}, \frac{p}{\delta}\right\}\end{array}$ & $\begin{array}{l}a^{2} \beta^{2}+r^{2} \\
+\quad b r \beta \quad> \\
\operatorname{emr} \beta \max \left\{\frac{1}{q}, \frac{p}{\delta}\right\} .\end{array}$ \\
\hline$E_{2}{ }^{n}$ & Infeasible. & - & - & - \\
\hline$E_{3}{ }^{n}$ & $\begin{array}{l}\frac{r-2 \beta x_{I 3}}{y_{I 3}} \\
<m<m^{[I]} .\end{array}$ & $\begin{array}{l}\frac{\left(r-2 \beta x_{I I 3}\right)\left(a+x_{I I 3}\right)^{2}}{a y_{I I 3}} \\
<m<m^{[I I]}\end{array}$ & $\begin{array}{l}\text { (i) } m_{[I I I]}< \\
m<m^{[I I I]}, \\
\text { (ii) } a>x_{I I I 3} .\end{array}$ & $\begin{array}{l}\text { (i) } m_{[I V]}< \\
m<m^{[I V]} \\
\text { (ii) } a>x_{I V 3} .\end{array}$ \\
\hline$E_{4}{ }^{n}$ & $\delta<\delta^{[I]}$ & $\begin{array}{l}\frac{\left(r-2 \beta x_{I I 4}\right)\left(a+x_{I I 4}\right)^{2}}{a p y_{I I 4}} \\
<m<m^{[I I 1]}\end{array}$ & $\begin{array}{l}(i) m_{[I I I 1]}< \\
m< \\
m^{[I I I 1]},(i i) a> \\
x_{I I I 4} .\end{array}$ & $\begin{array}{l}\text { (i) } m_{[I V 1]}< \\
m<m^{[I V 1]} \\
\text { (ii) } a>x_{I V 4} .\end{array}$ \\
\hline$E_{5}{ }^{n}$ & $\begin{array}{l}(i) \frac{r}{2 \beta}<x_{I 5}< \\
\mu^{[I]}, \\
(i i) h_{2}<\lambda< \\
\left(h_{2}+m\right), \\
(i i i) \delta>(\lambda- \\
\left.h_{2}\right) y_{I 5}+(p(\lambda+ \\
\left.\left.h_{1}\right)-2 h_{2}\right) z_{I 5}+ \\
e m p x_{I 5} .\end{array}$ & $\begin{array}{l}\text { (i) } a>x_{I I 5}, \\
\left(\text { ii) } h_{2}<\lambda<\right. \\
\lambda^{[I I]}, \\
\left(\text { iii) } \mu^{[I I]}<\right. \\
\beta<\mu^{[I I]}+ \\
h_{2} \frac{z_{I I 5}}{x_{I I 5}} .\end{array}$ & $\begin{array}{l}\text { (i) } a>x_{I I I 5}, \\
\text { (ii) } h_{2}<\lambda< \\
\lambda^{[I I I]}, \\
\left(\text { iii) } \mu^{[I I I]}<\right. \\
\beta<\mu^{[I I I]}+ \\
h_{2} \frac{z_{I I I 5}}{x_{I I I 5}} .\end{array}$ & 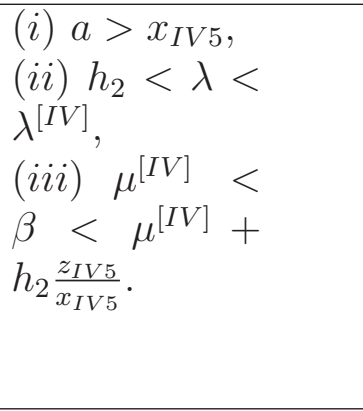 \\
\hline
\end{tabular}

Table 2: Comparison of the global stability conditions of the various Equilibria; $n$ denotes the model number. 


\begin{tabular}{|c|c|}
\hline $\begin{array}{l}\text { Interior } \\
\text { Equilibria }\end{array}$ & Conditions For Global Stability \\
\hline$E_{5}{ }^{I}$ & $\begin{array}{l}\text { (a) } \frac{\lambda+3 h_{1}}{2\left(\lambda-h_{2}\right)} \leq \min \left(\frac{\lambda+h_{1}+e m p}{2\left(\lambda-3 h_{2}+e m p\right)}, \frac{m p+2 \beta)}{2 m p}\right) \\
\text { (b) } \lambda>h_{2}\end{array}$ \\
\hline$E_{5}{ }^{I I}$ & $\begin{array}{l}\text { (i) } \beta>m \frac{p z_{I I 5}+y_{I I 5}}{a\left(a+x_{I I 5}\right)} \\
\text { (ii) } 4 h_{1}\left(\beta-m \frac{p z_{I I 5}+y_{I I 5}}{a\left(a+x_{I I 5}\right)}\right)>\left(\frac{m\left(a(1-e)+x_{I I 5}\right)}{a\left(a+x_{I I 5}\right)}\right)^{2}, \\
\text { (iii) }\left(\frac{p m(1-e)}{a+W}+\frac{e m p x_{I I 5}}{\left(a+x_{I I 5}\right)(a+W)}\right)\left(\frac{(1-e) m}{a+W}+\frac{e m p x_{I I 5}}{(a+W)\left(a+x_{I I 5}\right)}\right) \\
>4 \frac{\left(h_{1}+h_{2}\right)}{2}\left(\beta-\frac{p m z_{I I 5}+m y_{I I 5}}{a\left(a+x_{I I 5}\right)}\right), \\
\text { (iv) } h_{1}+h_{2}>2 h_{1} p .\end{array}$ \\
\hline$E_{5}{ }^{I I I}$ & $\begin{array}{l}\text { (i) } \beta>m \frac{\left(p z_{I I I 5}+y_{I I I 5}\right)\left(W+x_{I I I 5}\right)}{a^{2}\left(a^{2}+x_{I I I 5}^{2}\right)} \\
(i i) 4 h_{1}\left(\beta-m \frac{\left(p z_{I I I 5}+y_{I I I 5}\right)\left(W+x_{I I I 5}\right)}{a^{2}\left(a^{2}+x_{I I I 5}^{2}\right)}\right) \\
>\left(\frac{m\left((1-e)\left(a^{2}+x_{I I I 5}^{2}\right)+e m x_{I I I 5}\left(W+x_{I I I 5}\right)\right)}{a^{2}\left(a^{2}+x_{I I I 5}^{2}\right)}\right)^{2} \\
(\text { iii }) \frac{p m^{2}}{4}\left(\frac{(1-e)}{a^{2}+W^{2}}+\frac{e x_{I I I}^{2}}{\left(a^{2}+W^{2}\right)\left(a^{2}+x_{I I I 5}^{2}\right)}\right)^{2} \\
>\frac{\beta\left(h_{1}+h_{2}\right)}{2}-\frac{m x_{I I I 5}\left(y_{I I I 5}+p z_{I I I 5}\right)\left(h_{1}+h_{2}\right)}{2\left(a^{2}+W^{2}\right)\left(a^{2}+x_{I I I 5}^{2}\right)} \\
\text { (iv) } h_{1}+h_{2}>2 h_{1} p .\end{array}$ \\
\hline$E_{5}{ }^{I V}$ & $\begin{array}{l}\text { (i) } \beta>m \frac{\left(p z_{I V 5}+y_{I V 5}\right)\left(b+W+x_{I V 5}\right)}{a^{2}\left(a^{2}+b x_{I V 5}+x_{I V 5}^{2}\right)}, \\
\text { (ii) } 4 h_{1}\left(\beta-m \frac{\left(p z_{I V 5}+y_{I V 5}\right)\left(b+W+x_{I V 5}\right)}{a^{2}\left(a^{2}+b x_{I V 5}+x_{I V 5}^{2}\right)}\right) \\
>m^{2}\left(\frac{(1-e)\left(a^{2}+x_{I V 5}^{2}\right)+b x_{I V 5}+e x_{I V 5}\left(W+x_{I V 5}\right)}{\left.a^{2}\left(a^{2}+b x_{I V 5}+x_{I V 5}^{2}\right)\right)}\right)^{2}, \\
\text { (iii) } \frac{p m^{2}}{4}\left(\frac{(1-e)}{a^{2}+b W+W^{2}}+\frac{e x_{I V 5}\left(b+x_{I V 5}\right)}{\left(a^{2}+b W+W^{2}\right)\left(a^{2}+b x_{I V 5}+x_{I V 5}^{2}\right)}\right)^{2} \\
>\frac{\beta\left(h_{1}+h_{2}\right)}{2}-\frac{m\left(b+x_{I V 5}\right)\left(y_{I V 5}+p z_{I V 5}\right)\left(h_{1}+h_{2}\right)}{2\left(a^{2}+b W+W^{2}\right)\left(a^{2}+b x_{I V 5}+x_{I V 5}^{2}\right)}, \\
\text { (iv) } h_{1}+h_{2}>2 h_{1} p .\end{array}$ \\
\hline
\end{tabular}

Table 3: Comparison of the conditions for global stability of the interior equilibria $E_{5}{ }^{I}$, $E_{5}{ }^{I I}$, and $E_{5}^{I I I}$ and $E_{5}^{I V}$. 


\begin{tabular}{|c|c|c|c|}
\hline $\begin{array}{l}\text { Variable } / \\
\text { parameter }\end{array}$ & Units & Defination & Default value \\
\hline$x$ & $\begin{array}{l}\text { number per unit designated } \\
\text { area }\end{array}$ & prey density & $\cdots \cdots$ \\
\hline$y$ & $\begin{array}{l}\text { number per unit designated } \\
\text { area }\end{array}$ & density of healthy predator & $\cdots \cdots$ \\
\hline$z$ & $\begin{array}{l}\text { number per unit designated } \\
\text { area }\end{array}$ & density of infected predator & $\cdots \cdots$ \\
\hline$r$ & per day & growth rate of prey & 10.3 \\
\hline$\beta$ & per day & $\begin{array}{l}\text { intra-specific competition } \\
\text { rate of prey }\end{array}$ & 5 \\
\hline$a$ & per day & half saturation constant & 1 \\
\hline$b$ & per day & ecological parameter & 1.5 \\
\hline$p$ & per day & reduced factor & 0.001 \\
\hline$m$ & per day & predation rate & $\ldots \ldots$ \\
\hline$e$ & per day & conversion factor & 0.7 \\
\hline$h_{1}$ & per day & $\begin{array}{l}\text { intra and inter specific com- } \\
\text { petition rate of healthy } \\
\text { predator }\end{array}$ & $\cdots \cdots$ \\
\hline$h_{2}$ & per day & $\begin{array}{l}\text { intra and inter-specific com- } \\
\text { petition rate of infected } \\
\text { predator }\end{array}$ & $\ldots \ldots$ \\
\hline$\lambda$ & per day & $\begin{array}{l}\text { force of infection/contact } \\
\text { rate }\end{array}$ & 0.9 \\
\hline$q$ & per day & $\begin{array}{l}\text { death rate of healthy preda- } \\
\text { tor }\end{array}$ & 0.00005 \\
\hline$\delta$ & per day & $\begin{array}{l}\text { death rate of infected preda- } \\
\text { tor }\end{array}$ & 2 \\
\hline
\end{tabular}

Table 4: Variables and parameters used in the simulations. 


\section{References}

[1] D. Alstad, 2001, Basic Populas Models of Ecology, Prentice Hall, New Jersy.

[2] T. W. Anderson, Predator responses, prey refuges and density-dependent mortality of a marine fish, Ecology, 82(1),245-257, 2001.

[3] J. F. Andrews, A mathematical model for the continuous culture of microorganisms utilizing inhibitory substances, Biotechnology and Bioengineering, 10, 707-723, 1968.

[4] M. Haque, 2009, Ratio-dependent predator-prey models of interacting populations, Bulletin of Mathematical Biology, 71: 430-452.

[5] M. Haque, 2011, A detailed study of the Beddington-DeAngelis predator-prey model, Mathematical Bioscience, 234(1): 1-16.

[6] Mainul Haque, 2012, âĂIJExistence of complex patterns in the Beddington-DeAngelis predator-prey modelâĂ Mathematical Biosciences 239, 179âĂŞ190.

[7] May, R.M., 1974, Stability and Complexity in Model Ecosystem, Princeton University Press.

[8] Freedman, H. I., 1980, Deterministic Mathematical Models in Population Ecology, Marcel Debber., New York.

[9] M. Haque, S. Sarwardi, S. Preston and E. Venturino, 2011, Effect of delay in a LotkaVolterra type predator-prey model with a transmissible disease in the predator species, Mathematical Bioscience, 234(1):47-57.

[10] S. Belvisi, N. Tomatis, E. Venturino, Ecoepidemic models with identifiable infectives I: disease in the prey, Proceedings of the 10th International Conference on Computational and Mathematical Methods in Science and Engineering, CMMSE 2010, Almeria, Spain, June 27th-30th, 2010, v. 1, p. 188-198.

[11] V. Capasso, G. Serio, A generalization of the Kermack-McKendrick deterministic epidemic model, 1978, Mathematical Bioscience, 42, 43-61.

[12] J. B. Collings, 1997, The effects of the functional response on the bifurcation behaviour of a mite predator-prey interaction model, Journal of Mathematical Biology, 36, 149168.

[13] H. I. Freedman and G. S. K. Wolkowicz, 1986, Predator-prey systems with group defence: The paradox of enrichment revisited, Bulletin of Mathematical Biology, 48, 493-508.

[14] J. B. S. Haldane, Enzymes Longmans, London, 1930.

[15] M. Haque, E. Venturino, 2006, The role of transmissible diseases in Holling-Tanner predator-prey model, Theoretical Population Biology 70(3) 273-288.

[16] M. Haque, E. Venturino, 2009, Mathematical models of diseases spreading in symbiotic communities, in J.D. Harris, P.L. Brown (Editors), Wildlife: Destruction, Conservation and Biodiversity, NOVA Science Publishers, New York, 135-179. 
(i)

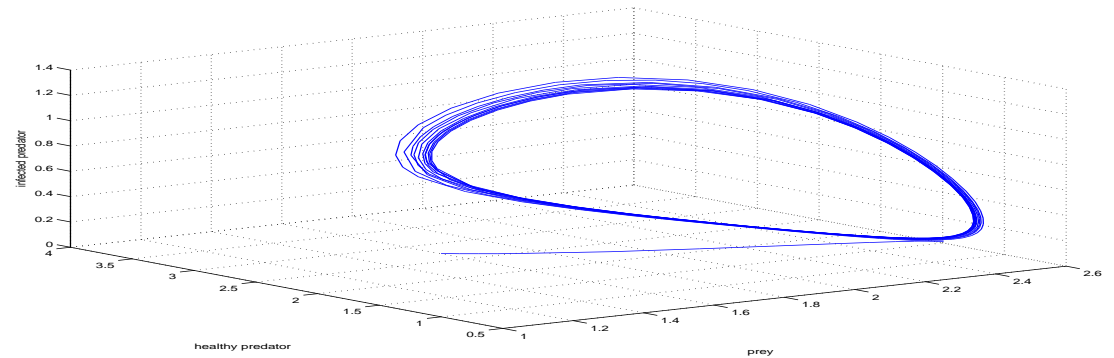

(ii)

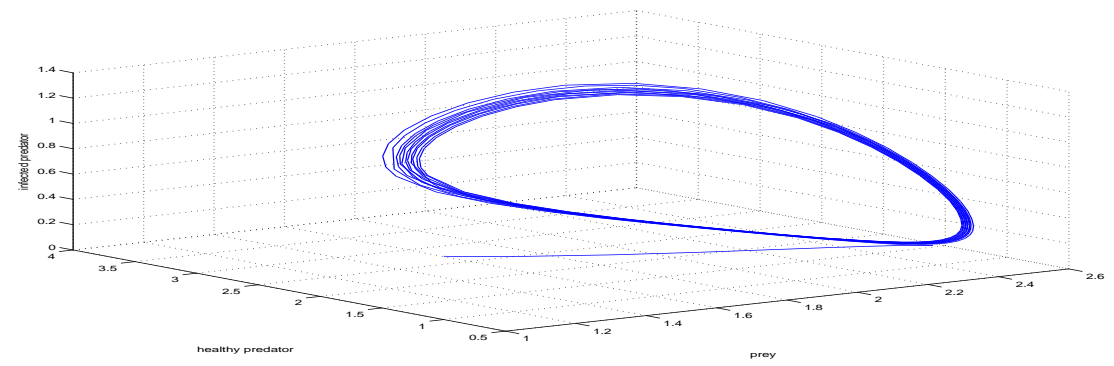

(iii)

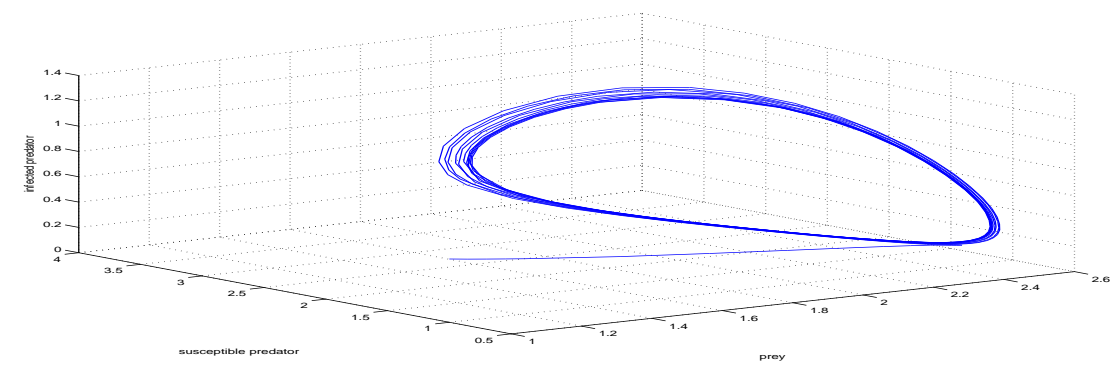

(iv)

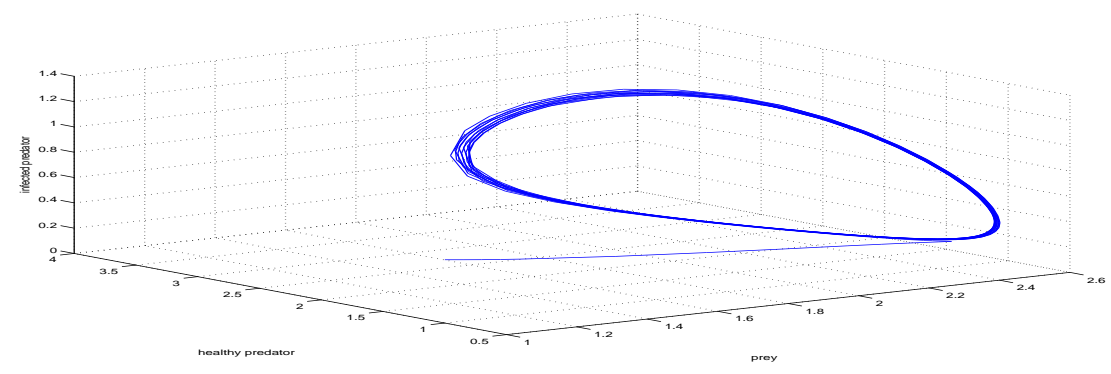

Figure 1: (i), (ii), (iii) and (iv) are the phase portrait of limit cycle oscillations of the system (2.4), (2.15), (2.27) with $b=0$, and (2.27) at the coexistence equilibria with fixed parameter values $\beta=1.2, a=2, h_{1}=0.005, h_{2}=0.002, \lambda=0.9, e=0.7, \delta=2$ and the set of values $r$ as $\{3.27,3.23,3.3,3.37\}$ the set of values $m$ as $\{2,2,2,3\}$ the set of values $p$ as $\{0.201,0.21,0.3,0.4\}$ and the set of values $q$ as $\{0.0009,0.00098,0.001,0.002\}$ respectively. 
[17] C. S. Holling, 1959, Some characteristics of simple types of predation and parasitism, Canadian Entomologist, 91, 385-398.

[18] J. C. Holmes and W. M. Bethel, 1972,Modification of intermediate host behavior by parasites, Zoolog. Journal Linnean Society, 51, Suppl. 1, 123-149.

[19] Hsieh, Y. and C. Hsiao, 2008, Predator-prey model with disease infection in both populations, Mathematical Medicine and Biology, 25, 247-266.

[20] E. Korpimaki, K. Norrdahl, 1991, Numerical and functional responses of kestrels, shorteared owls, and long-eared owls to vole densities, Ecology, 814-826.

[21] H. Malchow, S. Petrovskii, E. Venturino, 2008, Spatiotemporal patterns in Ecology and Epidemiology, CRC.

[22] K. Mischaikow and G. S. K. Wolkowicz, 1990, A predator-prey system involving group defense: A connection matrix approach, Nonlinear Analysis, 14, 955-969.

[23] Pal, R., Basu, D., Banerjee, M., 2009. Modelling of phytoplankton allelopathy with Monod-Haldane-type functional response - a mathematical study. Biosystems 95, 243253.

[24] R. E. Ricklefs, G. L. Miller, 2000, "Ecology", Fourth Edition, Williams and Wilkins Co, Inc.

[25] Ruan, S. and Xiao, D., 2000, Global analysis in a predator-prey system with nonmonotonic functional response, SIAM Journal on Applied Mathematics 61, 1445-1472.

[26] Saleh, K., 2011, Multiple Attractors Involving Chaos in Predator-prey Model with Non-monotonic Response Function, International Journal of Basic and Applied Sciences IJBAS-IJENS Vol: 11 No: 04, 116804-0909.

[27] W. Sokol, J. A. Howell, 1987, Kinetics of phenol oxidation by washed cell, Biotechnolgy and Bioengineering, 30, 921-927.

[28] J. S. Tener, Muskoxen, Queen's Printer, Ottawa, Canada, 1965.

[29] E. Venturino, 1994, The influence of diseases on Lotka-Volterra systems, Rocky Mountain Journal of Mathematics, vol. 24, p. 381-402.

[30] E. Venturino, 1995, Epidemics in predator-prey models: disease in the prey, in O. Arino, D. Axelrod, M. Kimmel, M. Langlais: Mathematical Population Dynamics: Analysis of Heterogeneity, Vol. one: Theory of Epidemics, Wuertz Publishing Ltd, Winnipeg, Canada, p. 381-393.

[31] E. Venturino, 2001, The effects of diseases on competing species, Mathematical Bioscience, 174, 111-131.

[32] E. Venturino, 2002, Epidemics in predator-prey models: disease in the predators, IMA Journal of Mathematics Applied in Medicine and Biology, 19, 185-205. 
[33] E. Venturino, 2006, On epidemics crossing the species barrier in interacting population models, Varahmihir Journal of Mathematical Science, 6(1), 247-263.

[34] E. Venturino, 2007, How diseases affect symbiotic communities, Mathematical Bioscience, 206, 11-30.

[35] G. S. K. Wolkowicz, Bifurcation analysis of a predator-prey system involving group defence, SIAM Journal of Applied Mathematics, 48, 592-606.

[36] T. Yano, Dynamics behaviour of the chemostat subject to substrate inhibition, Biotechnology and Bioengineering, 11, 139-153, 1969.

[37] E. C. Pielou, 1969, An introduction to mathematical ecology, John Wiley and Sons.

[38] A. D. Bazykin, A. I. Khibnik, and B. Krauskopf, 1998, Nonlinear dynamics of interacting populations, World Scientific.

[39] F.M.D. Gulland, 1995, The impact of infectious diseases on wild animal populations A review, in: B.T. Grenfell, A.P. Dobson (Eds.), Ecology and Infectious Diseases in Natural Populations, Cambridge University Press.

\section{Appendix}

\subsection{Appendix: Boundedness of the solution}

The boundedness of the solution (see [15] for details) can be shown in the following way. In fact, the prey are bounded since

$$
\limsup _{t \rightarrow+\infty} x(t)<\frac{r}{\beta}
$$

and further, introducing the total environment population $\Omega=x+y+z$, for an arbitrary $\eta>0$ the inequality

$$
\frac{d \Omega}{d t}+\eta \Omega \leq \phi
$$

follows, from which then all solutions of the system (2.4) starting in $R_{+}^{3}$ are confined in the region

$$
B=\left\{(x, y, z) \in R_{+}^{3}: \quad x+y+z \leq \frac{\phi}{\eta}+\epsilon \text { for any } \epsilon>0\right\}
$$

Remark. This argument applies also to the models (2.15), (2.27) with minor changes and will not be repeated.

The equilibria are the origin, always unstable, and five other points. Among these, note that $E_{2}{ }^{I}\left(0, y_{I 2}, z_{I 2}\right)$, cannot be feasible, because it should solve the equation $-\lambda z-q-h_{1}(y+z)=$ 0 , and this is not possible for both $y_{I 2} \geq 0, z_{I 2} \geq 0$. 


\subsection{Appendix A: Local stability analysis of model I}

For the proof of local stability, the concept of the second compound matrix is used. If $J_{I 5}=\left(\xi_{i j}\right), i, j=1,2,3$ represents the Jacobian of the model I, the second compound matrix is

$$
J^{[2]}=\left[\begin{array}{ccc}
\xi_{11}+\xi_{22} & \xi_{23} & -\xi_{13} \\
\xi_{32} & \xi_{11}+\xi_{33} & \xi_{12} \\
-\xi_{31} & \xi_{21} & \xi_{22}+\xi_{33}
\end{array}\right]
$$

Note that in our case the Jacobian has the following entries

$$
\begin{aligned}
& \xi_{11}=r-2 \beta x_{I 5}-m y_{I 5}-p m z_{I 5}, \quad \xi_{12}=-m x_{I 5}, \quad \xi_{13}=-p m x_{I 5}, \\
& \xi_{21}=e m y_{I 5}, \quad \xi_{22}=e m x_{I 5}-\lambda z_{I 5}-q-2 h_{1} y_{I 5}-h_{1} z_{I 5}, \quad \xi_{23}=-\lambda y_{I 5}-h_{1} y_{I 5}, \\
& \xi_{31}=e m p z_{I 5}, \quad \xi_{32}=\lambda z_{I 5}-h_{2} z_{I 5}, \quad \xi_{33}=\lambda y_{I 5}+e m p x_{I 5}-\delta-h_{2} y_{I 5}-2 h_{2} z_{I 5} .
\end{aligned}
$$

Proof of Proposition 1.. Let $D=\operatorname{diag}\left(x_{I 5}, y_{I 5}, z_{I 5}\right)$. The matrix $J^{[2]}\left(E_{5}{ }^{I}\right)$ is similar to $D J^{[2]}\left(E_{5}^{I}\right) D^{-1}=\left(d_{i j}\right)$, where $d_{11}=\xi_{11}+\xi_{22}, d_{22}=\xi_{11}+\xi_{33}, d_{33}=\xi_{22}+\xi_{33}$ and

$$
\begin{array}{ll}
d_{12}=\xi_{23} \frac{z_{I 5}}{y_{I 5}}, & d_{13}=-\xi_{13} \frac{z_{I 5}}{x_{I 5}},
\end{array}
$$

Hence $J^{[2]}\left(E_{5}{ }^{I}\right)$ and $D J^{[2]}\left(E_{5}{ }^{I}\right) D^{-1}$ have the same eigenvalues. Since the diagonal elements of $D J^{[2]}\left(E_{5}{ }^{I}\right) D^{-1}$ are negative, using Gershgorin's theorem the eigenvalues have negative real part if the matrix is row diagonally dominant. Set $\omega^{[I]}=\max \left\{g_{1}, g_{2}, g_{3}\right\}$, where $g_{k}=d_{k 1}+d_{k 2}+d_{k 3}, k=1,2,3$ and specifically

$$
\begin{array}{r}
g_{1}=r+(e m-2 \beta) x_{I 5}-y_{I 5}\left(m+2 h_{1}\right)-2 z_{I 5}\left(\lambda+h_{1}\right)-q, \\
g_{2}=\xi_{11}+\xi_{33}+\left(\lambda-h_{2}-m\right) y_{I 5}, \\
g_{3}=-e m p x_{I 5}+e m x_{I 5}+\xi_{22}+\xi_{33} .
\end{array}
$$

When (2.10) and (2.11) hold true then $\omega^{[I]}<0$, which implies diagonal dominance. Again with these assumptions we find $\xi_{11}<0, \xi_{12}<0, \xi_{13}<0, \xi_{21}>0, \xi_{22}<0, \xi_{23}<0, \xi_{31}>0$, $\xi_{32}>0, \xi_{33}<0$ and $\xi_{12}\left(\xi_{23} \xi_{31}-\xi_{21} \xi_{33}\right)<0$. Then we complete the proof by observing that

$$
\begin{aligned}
\operatorname{det}\left(J_{I 5}\right)= & =\xi_{11} \xi_{22} \xi_{33}-\xi_{11} \xi_{32} \xi_{23}+\xi_{13} \xi_{21} \xi_{32}-\xi_{13} \xi_{31} \xi_{22} \\
& +\xi_{12}\left(\xi_{23} \xi_{31}-\xi_{21} \xi_{33}\right)<0 .
\end{aligned}
$$




\subsection{Appendix B: The Jacobian matrix of model II}

The Jacobian of the model (2.15) is given by $J(x, y, z)=\left(\mu_{i j}\right)_{3 \times 3}$ where

$$
\begin{aligned}
\mu_{11} & =r-2 \beta x-\frac{m y}{a+x}+\frac{m x y}{(a+x)^{2}}-\frac{p m z}{a+x}+\frac{p m x z}{(a+x)^{2}}, \\
\mu_{12} & =-\frac{m x}{a+x}, \quad \mu_{13}=-\frac{p m x}{a+x}, \quad \mu_{21}=\frac{e m y}{a+x}-\frac{e m x y}{(a+x)^{2}}, \\
\mu_{22} & =\frac{e m x}{a+x}-\lambda z-q-h_{1}(y+z)-h_{1} y, \quad \mu_{23}=-\lambda y-h_{1} y, \\
\mu_{31} & =\frac{e m p z}{a+x}-\frac{e m p x z}{(a+x)^{2}}, \quad \mu_{32}=\lambda z-h_{2} z, \\
\mu_{33} & =\lambda y+\frac{e m p x}{a+x}-\delta-h_{2}(y+z)-h_{2} z .
\end{aligned}
$$

\subsection{Appendix C: The proof of Proposition 3 and 4}

\subsubsection{The proof of Proposition 3}

The proof of Proposition 3 is given by

Proof The signs of the entries of the Jacobian matrix $J_{I I 5}=\left(\phi_{i j}\right), i, j=1,2,3$ can be assessed as follows: $\phi_{I I 11}<0$ when $\beta>\mu^{[I I]} ; \phi_{22}<0 ; \phi_{33}<0$; and finally $\phi_{12}<0, \phi_{13}<0$, $\phi_{23}<0$, while $\phi_{32}>0$ for $\lambda>h_{2}, \phi_{21}>0, \phi_{31}>0$. It follows that

$$
\begin{aligned}
\operatorname{tr}\left(J_{I I 5}\right) & =-\beta x_{I I 5}+\frac{m\left(y_{I I 5}+p z_{I I 5}\right)}{\left(a+x_{I I 5}\right)^{2}}-h_{1} y_{I I 5}-h_{2} z_{I I 5}<0, \\
M_{I I 5} & \equiv\left(\phi_{11} \phi_{22}-\phi_{21} \phi_{12}\right)+\left(\phi_{11} \phi_{33}-\phi_{13} \phi_{31}\right)+\left(\phi_{22} \phi_{33}-\phi_{32} \phi_{23}\right)>0
\end{aligned}
$$

while

$$
\operatorname{det}\left(J_{I I 5}\right)=\phi_{11}\left(\phi_{22} \phi_{33}-\phi_{23} \phi_{32}\right)+\phi_{12}\left(\phi_{31} \phi_{23}-\phi_{21} \phi_{33}\right)<0
$$

if $\phi_{31} \phi_{23}>\phi_{21} \phi_{33}$, which follows from the condition $\lambda<\left(h_{2}-h_{1} p\right) p^{-1}$. Finally, we see that

$$
\begin{aligned}
C_{I I 5} \equiv & \operatorname{tr}\left(J_{I I 5}\right) M_{I I 5}-\operatorname{det}\left(J_{I I 5}\right) \\
= & \phi_{11}^{2}\left(\phi_{22}+\phi_{33}\right)+\phi_{22}{ }^{2}\left(\phi_{11}+\phi_{33}\right)+\phi_{33}^{2}\left(\phi_{11}+\phi_{22}\right)+2 \phi_{11} \phi_{22} \phi_{33} \\
& -\phi_{13}\left(\phi_{33} \phi_{31}+\phi_{21} \phi_{32}\right)-\phi_{23} \phi_{32}\left(\phi_{33}-\phi_{11}\right)-\phi_{12} \phi_{31} \phi_{23} \\
& -\phi_{11}\left(\phi_{32} \phi_{23}+\phi_{31} \phi_{13}+\phi_{12} \phi_{21}\right)-\phi_{22} \phi_{32} \phi_{23}-\phi_{22} \phi_{12} \phi_{21}<0
\end{aligned}
$$

if $\phi_{33}-\phi_{11}<0$, and $\phi_{33} \phi_{31}+\phi_{21} \phi_{32}<0$ which both follow from the conditions $(2.22)$. Hence the Routh-Hurwitz conditions hold so that $E_{5}{ }^{I I}$ is locally asymptotically stable. 


\subsubsection{The proof of Proposition 4}

Proof of proposition 4. Let $R_{x}^{3}=\left[U^{T} \equiv(x, y, z) \in R_{x}^{+}, x \geq 0, y \geq 0, z \geq 0\right]$ and consider the scalar function $L_{I I}: R_{x}^{3} \rightarrow R, \frac{d L_{I I}}{d t}=\sum_{k=1}^{3} \frac{d}{d t} L_{I I k}(x, y, z)$, given by

$$
L_{I I}(t)=\left(x-x_{I I 5}-x_{I I 5} \ln \frac{x}{x_{I I 5}}\right)+\left(y-y_{I I 5}-y_{I I 5} \ln \frac{y}{y_{I I 5}}\right)+\left(z-z_{I I 5}-z_{I I 4} \ln \frac{z}{z_{I I 5}}\right)
$$

Its derivative of along solutions of the system (2.15) gives the quadratic form

$$
\frac{d L_{I I}}{d t}=-U^{T} M_{I} U
$$

where

$$
M_{I I}=\left[\begin{array}{ccc}
A_{I I} & H_{I I} & G_{I I} \\
H_{I I} & B_{I I} & F_{I I} \\
G_{I I} & F_{I I} & C_{I I}
\end{array}\right],
$$

and whose elements are

$$
\begin{array}{r}
A_{I I}=\beta-\frac{m\left(p z_{I I 5}+y_{I I 5}\right)}{\left(a+x_{I I 5}\right)(a+x)}, \quad H_{I I}=\frac{m}{2}\left(\frac{(1-e)}{a+x}+\frac{e x_{I I 5}}{(a+x)\left(a+x_{I I 5}\right)}\right), \quad B_{I I}=h_{1}, \\
F_{I I}=\frac{h_{1}+h_{2}}{2}, \quad G_{I I}=\frac{m p(1-e)}{2(a+x)}+\frac{e m p x_{I I 5}}{2(a+x)\left(a+x_{I I 5}\right)} \quad C_{I I}=h_{2} .
\end{array}
$$

In fact,

$$
\begin{aligned}
\frac{d L_{I I 1}}{d t}= & \left(x-x_{I I 5}\right)\left(\beta x_{I I 5}+\frac{m y_{I I 5}}{a+x_{I I 5}}+\frac{p m z_{I I 5}}{a+x_{I I 5}}-\beta x-\frac{m y}{a+x}-\frac{p m z}{a+x}\right) \\
= & \left(x-x_{I I 5}\right)\left(-\beta\left(x-x_{I I 5}\right)-m \frac{y-y_{I I 5}}{a+x}-p m \frac{z-z_{I I 5}}{a+x}+m \frac{y_{I I 5}\left(x-x_{I I 5}\right)}{\left(a+x_{I I 5}\right)(a+x)}\right. \\
& \left.+p m z_{I I 5} \frac{x-x_{I I 5}}{\left(a+x_{I I 5}\right)(a+x)}\right) ; \\
\frac{d L_{I I 2}}{d t}= & \left(y-y_{I I 5}\right)\left(\frac{e m x}{a+x}-\lambda z-q-h_{1}(y+z)\right) \\
= & \left(y-y_{I I 5}\right)\left(\frac{e m\left(x-x_{I I 5}\right)}{a+x}-\left(\lambda+h_{1}\right)\left(z-z_{I I 5}\right)-h_{1}\left(y-y_{I I 5}\right)-\frac{e m x_{I I 5}\left(x-x_{I I 5}\right)}{(a+x)\left(a+x_{I I 5}\right)}\right) ; \\
\frac{d L_{I I 3}=}{d t} & \left(z-z_{I I 5}\right)\left(\lambda y+\frac{e m p x}{a+x}-\delta-h_{2}(y+z)\right) \\
= & \left(z-z_{I I 5}\right)\left(\frac{e m p\left(x-x_{I I 5}\right)}{a+x}+\left(\lambda-h_{2}\right)\left(y-y_{I I 5}\right)-h_{2}\left(z-z_{I I 5}\right)-\frac{e m p x_{I I 5}\left(x-x_{I I 5}\right)}{(a+x)\left(a+x_{I I 5}\right)}\right) .
\end{aligned}
$$

Thus, if the matrix $M_{I I}$ is positive definite then $\frac{d L_{I I}}{d t}<0$. For that, we require all principal 
minors of $M_{I I}$ to be positive, namely

$$
\begin{aligned}
P_{I I 1} & \equiv A_{I I}=\beta-\frac{p m z_{I I 5}+m y_{I I 5}}{\left(a+x_{I I 5}\right)(a+x)}, \quad P_{I I 2} \equiv A_{I I} B_{I I}-H_{I I}^{2}, \\
P_{I I 3} & \equiv A_{I I} B_{I I} C_{I I}+2 F_{I I} G_{I I} H_{I I}-A_{I I} F_{I I}^{2}-B_{I I} G_{I I}{ }^{2}-C_{I I} H_{I I}{ }^{2} \\
& =C_{I I}\left(A_{I I} B_{I I}-H_{I I}{ }^{2}\right)+G_{I I}\left(F_{I I} H_{I I}-B_{I I} G_{I I}\right)+F_{I I}\left(G_{I I} H_{I I}-A_{I I} F_{I I}\right) .
\end{aligned}
$$

Now differentiating $P_{I I 1}$ when $x>0$, we find

$$
A_{I I}^{\prime}(x)=\frac{p m z_{I I 5}+m y_{I I 5}}{\left(a+x_{I I 5}\right)} \frac{1}{(a+x)^{2}}>0
$$

so that $A_{I I}^{\prime}(x)$ is a monotonic increasing function. Therefore by $(2.23)$ we get $A_{I I}(x)>$ $A(0)=\beta-\left(p m z_{I I 5}+m y_{I I 5}\right)\left[a\left(a+x_{I I 5}\right)\right]^{-1}>0$ and thus $P_{I I 1}=A_{I I}>0$. Next by $(2.24)$ we find

$$
P_{I I 2}=\left(\beta-\frac{p m z_{I I 5}+m y_{I I 5}}{\left(a+x_{I I 5}\right)(a+x)}\right) h_{1}-\frac{m^{2}}{4}\left(\frac{1-e}{a+x}+\frac{e x_{I I 5}}{(a+x)\left(a+x_{I I 5}\right)}\right)^{2}>0 .
$$

Obviously $F_{I I}>0$. For $P_{I I 3}$, by $(2.25)$, we have

$$
\begin{aligned}
G_{I I} H_{I I}-A_{I I} F_{I I}= & {\left[\frac{m p(1-e)}{2(a+x)}+\frac{e m p x_{I I 5}}{2(a+x)\left(a+x_{I I 5}\right)}\right] \frac{m}{2}\left[\frac{1-e}{a+x}+\frac{e x_{I I 5}}{2(a+x)\left(a+x_{I I 5}\right)}\right] } \\
& -\left[\beta-\frac{p m z_{I I 5}+m y_{I I 5}}{\left(a+x_{I I 5}\right)(a+x)}\right] \frac{h_{1}+h_{2}}{2} \\
& >\left[\frac{p m(1-e)}{a+W}+\frac{e m p x_{I I 5}}{\left(a+x_{I I 5}\right)(a+W)}\right]\left[\frac{(1-e) m}{a+W}\right. \\
& \left.+\frac{e m p x_{I I 5}}{(a+W)\left(a+x_{I I 5}\right)}\right]-4 \frac{h_{1}+h_{2}}{2}\left[\beta-\frac{p m z_{I I 5}+m y_{I I 5}}{a\left(a+x_{I I 5}\right)}\right]>0,
\end{aligned}
$$

so that $F_{I I}\left(G_{I I} H_{I I}-A_{I I} F_{I I}\right)>0$. Again for $P_{I I 3}$ by $(2.26)$ we have

$$
F_{I I} H_{I I}-B_{I I} G_{I I}=\left[\frac{m(1-e)}{4(a+x)}+\frac{e m x_{I I 5}}{4(a+x)\left(a+x_{I I 5}\right)}\right]\left[h_{1}+h_{2}-2 h_{1} p\right]>0 .
$$

so that also $G_{I I}\left(F_{I I} H_{I I}-B_{I I} G_{I I}\right)>0$. Combining these results it follows that $P_{I I 3}>$ 0 . Hence the symmetric matrix $M_{I I}$ is positive definite, implying $\frac{d L_{I I}}{d t}<0$ along the trajectories and $\frac{d L_{I I}}{d t}=0$, when $(x, y, z)=\left(x_{I I 5}, y_{I I 5}, z_{I I 5}\right)$. Thus, $L_{I I}$ is a Lyapunov function and global stability follows. 


\subsection{Appendix D: The Jacobian of the model (2.27)}

The Jacobian of the system (2.27) is given by $J(x, y, z)=\left(\kappa_{i j}\right)_{3 \times 3}$ with entries given by $\kappa_{23}=-\lambda y-h_{1} y, \kappa_{32}=\lambda z-h_{2} z$ and

$$
\begin{aligned}
\kappa_{11} & =r-2 \beta x-\frac{m(y+p z)}{x^{2}+b x+a^{2}}+\frac{m x(y+p z)(2 x+b)}{\left(x^{2}+b x+a^{2}\right)^{2}}, \\
\kappa_{13} & =-\frac{p m x}{x^{2}+b x+a^{2}}, \quad \kappa_{21}=\frac{e m y}{x^{2}+b x+a^{2}}-\frac{e m x y(2 x+b)}{\left(x^{2}+b x+a^{2}\right)^{2}}, \\
\kappa_{22} & =\frac{e m x}{x^{2}+b x+a^{2}}-\lambda z-q-h_{1}(y+z)-h_{1} y, \\
\kappa_{31} & =\frac{e m p z}{x^{2}+b x+a^{2}}-\frac{e m p x z(2 x+b)}{\left(x^{2}+b x+a^{2}\right)^{2}}, \quad \kappa_{12}=-\frac{m x}{x^{2}+b x+a^{2}}, \\
\kappa_{33} & =\lambda y+\frac{e m p x}{x^{2}+b x+a^{2}}-\delta-h_{2}(y+z)-h_{2} z,
\end{aligned}
$$

\subsection{Appendix E: The proofs of the propositions 5, 6 and 7}

\subsubsection{The proof of the proposition 5:}

Proof. The Jacobian is now a full matrix $J_{I V 5}=\left(\bar{\phi}_{i j}\right), i, j=1,2,3$ If we assume $a>x_{I V 5}$, $\beta>\mu^{[I V]}$, and $\lambda>h_{2}$ the components of the Jacobian are all negative but for $\bar{\phi}_{32}, \bar{\phi}_{21}, \bar{\phi}_{31}$. It then follows that $\operatorname{tr}\left(J_{I V 5}\right)<0, M_{I V 5} \equiv\left(\bar{\phi}_{11} \bar{\phi}_{22}-\bar{\phi}_{21} \bar{\phi}_{12}\right)+\left(\bar{\phi}_{11} \bar{\phi}_{33}-\bar{\phi}_{13} \bar{\phi}_{31}\right)+\left(\bar{\phi}_{22} \bar{\phi}_{33}-\right.$ $\left.\bar{\phi}_{32} \bar{\phi}_{23}\right)>0$ and

$$
\begin{aligned}
\operatorname{det}\left(J_{I V 5}\right)= & \bar{\phi}_{11}\left(\bar{\phi}_{22} \bar{\phi}_{33}-\bar{\phi}_{23} \bar{\phi}_{32}\right)+\bar{\phi}_{12}\left(\bar{\phi}_{31} \bar{\phi}_{23}-\bar{\phi}_{21} \bar{\phi}_{33}\right) \\
& +\bar{\phi}_{13}\left(\bar{\phi}_{32} \bar{\phi}_{21}-\bar{\phi}_{31} \bar{\phi}_{22}\right) \\
C_{I V 5} \equiv & \operatorname{tr}\left(J_{I V 5}\right) M_{I V 5}-\operatorname{det}\left(J_{I V 5}\right) \\
= & \bar{\phi}_{11}^{2}\left(\bar{\phi}_{22}+\bar{\phi}_{33}\right)+\bar{\phi}_{22}^{2}\left(\bar{\phi}_{11}+\bar{\phi}_{33}\right)+\bar{\phi}_{33}^{2}\left(\bar{\phi}_{11}+\bar{\phi}_{22}\right)+2 \bar{\phi}_{11} \bar{\phi}_{22} \bar{\phi}_{33} \\
& -\bar{\phi}_{13}\left(\bar{\phi}_{33} \bar{\phi}_{31}+\bar{\phi}_{21} \bar{\phi}_{32}\right)-\bar{\phi}_{23} \bar{\phi}_{32}\left(\bar{\phi}_{33}-\bar{\phi}_{11}\right)-\bar{\phi}_{12} \bar{\phi}_{31} \bar{\phi}_{23} \\
& -\bar{\phi}_{11}\left(\bar{\phi}_{32} \bar{\phi}_{23}+\bar{\phi}_{31} \bar{\phi}_{13}+\bar{\phi}_{12} \bar{\phi}_{21}\right)-\bar{\phi}_{22} \bar{\phi}_{32} \bar{\phi}_{23}-\bar{\phi}_{22} \bar{\phi}_{12} \bar{\phi}_{21} .
\end{aligned}
$$

Now, $\operatorname{det}\left(J_{I V 5}\right)<0$ if the second term in its expression is negative, i.e., if $\bar{\phi}_{31} \bar{\phi}_{23}>\bar{\phi}_{21} \bar{\phi}_{33}$. But this follows from the first condition (2.34). Finally, $C_{I V 5}<0$ if $\left(\bar{\phi}_{33}-\bar{\phi}_{11}\right)<0$ and $\left(\bar{\phi}_{33} \bar{\phi}_{31}+\bar{\phi}_{21} \bar{\phi}_{32}\right)<0$ which also follows from the conditions $(2.34)$. Hence the RouthHurwitz conditions hold, and local asymptotic stability follows.

\subsubsection{The proof of the proposition 6}

Proof. Again, consider the scalar function $L_{I V}: R_{x}^{3} \rightarrow R$

$$
L_{I V}(t)=\left(x-x_{I V 5}-x_{I V 5} \ln \frac{x}{x_{I V 5}}\right)+\left(y-y_{I V 5}-y_{I V 5} \ln \frac{y}{y_{I V 5}}\right)+\left(z-z_{I V 5}-z_{I V 5} \ln \frac{z}{z_{I V 5}}\right)
$$


with derivative along solution trajectories of (2.27) which are given by

$$
\begin{aligned}
\frac{d L_{I V 1}}{d t}= & \left(x-x_{I V 5}\right)^{2}\left(-\beta-\frac{m y_{I V 5}\left(b+x+x_{I V 5}\right)}{\left(a^{2}+b x+x^{2}\right)\left(a^{2}+b x_{I V 5}+x_{I V 5}^{2}\right)}\right. \\
& \left.+\frac{p m z_{I V 5}\left(b+x+x_{I V 5}\right)}{\left(a^{2}+b x+x^{2}\right)\left(a^{2}+b x_{I V 5}+x_{I V 5}^{2}\right)}\right)-\frac{m\left(y-y_{I V 5}\right)\left(x-x_{I V 5}\right)}{\left(a^{2}+b x+x^{2}\right)} \\
& -\frac{p m\left(z-z_{I V 5}\right)\left(x-x_{I V 5}\right)}{\left(a^{2}+b x+x^{2}\right)} \\
\frac{d L_{I V 2}}{d t}= & \left(y-y_{I V 5}\right)\left(\frac{e m\left(x-x_{I V 5}\right)}{a^{2}+b x+x^{2}}-\left(\lambda+h_{1}\right)\left(z-z_{I V 5}\right)-h_{1}\left(y-y_{I V 5}\right)\right. \\
\frac{d L_{I V 3}}{d t}= & \left.-\frac{e m x_{I V 5}\left(x-x_{I V 5}\right)\left(b+x+x_{I V 5}\right)}{\left(a^{2}+b x+x^{2}\right)\left(a^{2}+b x_{I V 5}+x_{I V 5}^{2}\right)}\right) \\
& \left.-\frac{e m p x_{I V 5}\left(x-x_{I V 5}\right)\left(b+x+x_{I V 5}\right)}{\left(a^{2}+b x+x^{2}\right)\left(a^{2}+b x_{I V 5}+x_{I V 5}^{2}\right)}\right)
\end{aligned}
$$

and which can be written in matrix notation as

$$
\frac{d L_{I V}}{d t}=-U^{T} M_{I V} U
$$

where the symmetric quadratic form $M_{I V}$ is given by the expression (4.2) with obvious changes in the subscripts. The entries now are

$$
\begin{array}{r}
A_{I V}=\beta-\frac{m\left(p z_{I V 5}+y_{I V 5}\right)\left(b+x+x_{I V 5}\right)}{\left(a^{2}+b x+x^{2}\right)\left(a^{2}+b x_{I V 5}+x_{I V 5}^{2}\right)}, \quad B_{I V}=h_{1}, \quad C_{I V}=h_{2}, \\
H_{I V}=\frac{m}{2}\left(\frac{1-e}{a^{2}+b x+x^{2}}+\frac{e x_{I V 5}\left(b+x+x_{I V 5}\right)}{\left(a^{2}+b x+x^{2}\right)\left(a^{2}+b x_{I V 5}+x_{I V 5}^{2}\right)}\right), \\
F_{I V}=\frac{h_{1}+h_{2}}{2}, \quad G_{I V}=\frac{m p(1-e)}{2\left(a^{2}+b x+x^{2}\right)}+\frac{e m p x_{I V 5}\left(b+x+x_{I V 5}\right)}{2\left(a^{2}+b x+x^{2}\right)\left(a^{2}+b x_{I V 5}+x_{I V 5}^{2}\right)}
\end{array}
$$

Its principal minors can be shown to be all positive. In fact

$$
P_{I V 1}=A_{I V}(x)=\beta-\frac{m\left(p z_{I V 5}+y_{I V 5}\right)\left(b+x+x_{I V 5}\right)}{\left(a^{2}+b x_{I V 5}+x_{I V 5}^{2}\right)\left(a^{2}+b x+x^{2}\right)}>\beta-\frac{m\left(p z_{I V 5}+y_{I V 5}\right)\left(b+W+x_{I V 5}\right)}{\left(a^{2}+b x_{I V 5}+x_{I V 5}^{2}\right) a^{2}},
$$

therefore by the first $(2.35), A_{I V}(x)>0$. By (2.36)

$$
\begin{aligned}
P_{I V 2}= & A_{I V} B_{I V}-H_{I V}{ }^{2}=\left(\beta-\frac{m\left(p z_{I V 5}+y_{I V 5}\right)\left(b+x+x_{I V 5}\right)}{\left(a^{2}+b x_{I V 5}+x_{I V 5}^{2}\right)\left(a^{2}+b x+x^{2}\right)}\right) h_{1} \\
& -\frac{m^{2}}{4}\left(\frac{(1-e)}{a^{2}+b x+x^{2}}+\frac{e x_{I V 5}\left(b+x+x_{I V 5}\right)}{\left(a^{2}+b x+x^{2}\right)\left(a^{2}+b x_{I V 5}+x_{I V 5}^{2}\right)}\right)^{2}>0 .
\end{aligned}
$$


Clearly $F_{I V}>0$. For $P_{I V 3}$, we have by $(2.37)$,

$$
\begin{array}{r}
G_{I V} H_{I V}-A_{I V} F_{I V}>-\frac{\beta\left(h_{1}+h_{2}\right)}{2}+\frac{m\left(b+x_{I V 5}\right)\left(y_{I V 5}+p z_{I V 5}\right)\left(h_{1}+h_{2}\right)}{2\left(a^{2}+b W+W^{2}\right)\left(a^{2}+b x_{I V 5}+x_{I V 5}^{2}\right)} \\
+\frac{p m^{2}}{4}\left(\frac{1-e}{a^{2}+b W+W^{2}}+\frac{e x_{I V 5}\left(b+x_{I V 5}\right)}{\left.a^{2}+b W+W^{2}\right)\left(a^{2}+b x_{I V 5}+x_{I V 5}^{2}\right)}\right)^{2}>0
\end{array}
$$

and $F_{I V}\left(G_{I V} H_{I V}-A_{I V} F_{I V}\right)>0$. Further for $P_{I V 3}$, we have by the third (2.35)

$$
\begin{aligned}
& F_{I V} H_{I V}-B_{I V} G_{I V} \\
& =\left[m(1-e)+\frac{e m x_{I V 5}\left(b+x+x_{I V 5}\right)}{\left(a^{2}+b x_{I V 5}+x_{I V 5}^{2}\right)}\right] \frac{h_{1}+h_{2}-2 h_{1} p}{4\left(a^{2}+b x+x^{2}\right)}>0
\end{aligned}
$$

and using $G_{I V}>0$ we find $G_{I V}\left(F_{I V} H_{I V}-B_{I V} G_{I V}\right)>0$, so that combining these results $P_{I V 3}>0$. Hence $M_{I V}$ is positive definite, the derivative of $L_{I V}$ is negative along the trajectories and vanishes at $E_{I V 5}$. Thus, $L_{I V}$ is a Lyapunov function and global stability follows.

\subsubsection{The proof of the proposition 7}

Proof. The characteristic equation of the system $(2.27)$ at $E_{5}^{I V}\left(x_{I V 5}, y_{I V 5}, z_{I V 5}\right)$ is

$$
\hat{\omega}^{3}+K_{1} \hat{\omega}^{2}+K_{2} \hat{\omega}+K_{3}=0,
$$

where $K_{1} \equiv-\operatorname{tr}\left(J_{I V 5}\right), K_{2} \equiv M_{I V 5}, K_{3} \equiv-\operatorname{det}\left(J_{I V 5}\right)$ and $K_{1} K_{2}-K_{3} \equiv-C_{I V 5}$. Hopf bifurcation will occur if and only if there exists $m=m_{c r}$ such that $(i) K_{1}\left(m_{c r}\right) K_{2}\left(m_{c r}\right)=$ $K_{3}\left(m_{c r}\right)$ with $K_{1}\left(m_{c r}\right), K_{2}\left(m_{c r}\right), K_{3}\left(m_{c r}\right)>0$ and $\left.(i i) \frac{d}{d m}(\operatorname{Re}(\hat{\omega}(m)))\right|_{m=m_{c r}} \neq 0$. Now when $m=m_{c r}, K_{1} K_{2}=K_{3}$ with $K_{1}, K_{2}, K_{3}>0$. The characteristic equation is given by

$$
\left(\hat{\omega}^{2}+K_{2}\right)\left(\hat{\omega}+K_{1}\right)=0
$$

with roots $\hat{\omega}_{1}=i \sqrt{K_{2}}, \hat{\omega}_{2}=-i \sqrt{K_{2}}$ and $\hat{\omega}_{3}=-K_{1}$, so that there exists a pair of purely imaginary eigenvalues and a strictly negative real eigenvalue. For $m$ in a neighbourhood of $m_{c r}$, the roots have the form $\hat{\omega}_{1}\left(m_{c r}\right)=q_{1}(m)+i q_{2}(m), \hat{\omega}_{2}=q_{1}(m)-i q_{2}(m)$, and $\hat{\omega}_{3}(m)=$ $-q_{3}(m)$ where $q_{1}(m), q_{2}(m)$, and $q_{3}(m)$ are real. Next, we shall verify the transversality condition

$$
\left.\frac{d}{d m}\left(\operatorname{Re}\left(\hat{\omega}_{j}(m)\right)\right)\right|_{m=m_{c r}} \neq 0, \quad \mathrm{j}=1,2
$$

Substituting $\hat{\omega}(m)=q_{1}(m)+i q_{2}(m)$ into the characteristic equation and taking the derivative we have

$$
\hat{\Psi}(m) \dot{q}_{1}(m)-\hat{\Phi}(m) \dot{q}_{2}(m)+\hat{\Theta}(m)=0, \quad \hat{\Phi}(m) \dot{q}_{1}(m)+\hat{\Psi}(m) \dot{q}_{2}(m)+\hat{\Gamma}(m)=0
$$


where

$$
\begin{aligned}
\hat{\Psi}(m) & =3\left(q_{1}(m)\right)^{2}+2 K_{1}(m) q_{1}(m)+K_{2}(m)-3\left(q_{2}(m)\right)^{2}, \\
\hat{\Phi}(m) & =6 q_{1}(m) q_{2}(m)+2 K_{1}(m) q_{2}(m), \\
\hat{\Theta}(m) & =\left(q_{1}(m)\right)^{2} \dot{K}_{1}(m)+q_{1}(m) \dot{K}_{2}(m) \\
& +\dot{K}_{3}(m)-\dot{K}_{1}(m)\left(q_{2}(m)\right)^{2},
\end{aligned}
$$

and

$$
\hat{\Gamma}(m)=2 q_{1}(m) q_{2}(m) \dot{K}_{1}(m)+\dot{K}_{2}(m) q_{2}(m)
$$

Now

$$
\begin{aligned}
\left.\frac{d}{d m}\left(R e\left(\hat{\omega}_{j}(m)\right)\right)\right|_{m=m_{c r}} & =-\left.\frac{(\hat{\Phi} \hat{\Gamma}+\hat{\Psi} \hat{\Theta})}{\hat{\Psi}^{2}+\hat{\Phi}^{2}}\right|_{m}=m_{c r} \\
& \neq 0 \\
\text { and } q_{3}\left(m_{c r}\right) & =-K_{1}\left(m_{c r}\right) \\
& \neq 0 .
\end{aligned}
$$

This completes the proof.

\subsection{Appendix F: $\omega_{i j}$ 's}

The $\omega_{i j}$ 's are given by

$$
\begin{aligned}
\omega_{11} & =r-2 \beta x_{I V 3}-\frac{m y_{I V 3}}{x_{I V 3}{ }^{2}+b x_{I V 3}+a^{2}}+\frac{m x_{I V 3} y_{I V 3}\left(2 x_{I V 3}+b\right)}{\left(x_{I V 3}{ }^{2}+b x_{I V 3}+a^{2}\right)^{2}}, \\
\omega_{12} & =-\frac{m x_{I V 3}}{x_{I V 3}{ }^{2}+b x_{I V 3}+a^{2}}, \\
\omega_{21} & =\frac{e m y_{I V 3}}{x_{I V 3}{ }^{2}+b x_{I V 3}+a^{2}}-\frac{e m x_{I V 3} y_{I V 3}\left(2 x_{I V 3}+b\right)}{\left(x_{I V 3}{ }^{2}+b x_{I V 3}+a^{2}\right)^{2}}, \\
\omega_{22} & =\frac{e m x_{I V 3}}{x_{I V 3}{ }^{2}+b x_{I V 3}+a^{2}}-q-2 h_{1} y_{I V 3} .
\end{aligned}
$$

\subsection{The coefficients $A_{i}$ 's and positive root conditions}

\subsubsection{The $A_{i}$ 's}

The $A_{i}$ 's are defined below: 


$$
\begin{aligned}
A_{5}= & \lambda \beta h_{1}-h_{2} \beta \lambda+\lambda^{2} \beta, \\
A_{4}= & h_{2} r \lambda-2 h_{2} \beta b \lambda+2 \lambda^{2} \beta b-\lambda^{2} r+2 \lambda \beta b h_{1}-\lambda r h_{1}, \\
A_{3}= & \lambda^{2} b^{2} \beta-2 \lambda^{2} r b-2 \lambda r b h_{1}+\lambda b^{2} \beta h_{1}+2 h_{2} r b \lambda+2 \lambda \beta a^{2} h_{1} \\
& +2 \lambda^{2} \beta a^{2}-2 h_{2} \beta a^{2} \lambda-h_{2} b^{2} \beta \lambda, \\
A_{2}= & \delta m \lambda-2 \lambda^{2} r a^{2}-\delta m h_{1} p+\delta m h_{1}+2 \lambda^{2} b \beta a^{2}-h_{2} q m \\
& -\lambda^{2} b^{2} r-2 \lambda r a^{2} h_{1}+h_{2} p q m-\lambda b^{2} r h_{1}+2 \lambda b \beta a^{2} h_{1} \\
& -2 h_{2} b \beta a^{2} \lambda+2 h_{2} r a^{2} \lambda-\lambda p q m+h_{2} b^{2} r \lambda, \\
A_{1}= & h_{2} p q b m+\lambda a^{4} \beta h_{1}-2 \lambda b r a^{2} h_{1}-h_{2} a^{4} \beta \lambda+2 h_{2} b r a^{2} \lambda \\
& -h_{2} p e m^{2}-\delta b m h_{1} p-\lambda p q b m-h_{2} q b m+e m^{2} p^{2} h_{1} \\
& -e m^{2} p h_{1}-2 \lambda^{2} b r a^{2}+h_{2} e m^{2}+\delta b m h_{1}+\lambda^{2} a^{4} \beta+\delta b m \lambda, \\
A_{0}= & h_{2} p q a^{2} m-\lambda p q a^{2} m-\delta a^{2} m h_{1} p-\lambda^{2} a^{4} r+\delta a^{2} m h_{1} \\
& -\lambda a^{4} r h_{1}-h_{2} q a^{2} m+\delta a^{2} m \lambda+h_{2} a^{4} r \lambda .
\end{aligned}
$$

\subsubsection{Condition for positive root of (2.33)}

We find sufficient conditions for it to have a positive real root of the equation (2.33). Assume that there are two pairs of complex conjugate roots $\omega_{1}, \omega_{1}{ }^{*}$ and $\eta_{1}, \eta_{1}{ }^{*}$. Each pair arises from a suitable quadratic with negative discriminant, respectively $\left(I-\omega_{1}\right)\left(I-\omega_{1}^{*}\right)=$ $I^{2}-2 \operatorname{Re}\left(\omega_{1}\right) I+\left|\omega_{1}\right|^{2},\left(I-\eta_{1}\right)\left(I-\eta_{1}{ }^{*}\right)=I^{2}-2 \operatorname{Re}\left(\eta_{1}\right) I+\left|\eta_{1}\right|^{2}$. Let $m_{1}=-2 \operatorname{Re}\left(\omega_{1}\right)$, $n_{1}=\left|\omega_{1}\right|^{2}>0, u=-2 R e\left(\eta_{1}\right)$ and $v=\left|\eta_{1}\right|^{2}>0$. Equation (2.33) will then have the following factorization, where $\alpha \equiv I_{5}$ is the sought real root,

$$
\begin{gathered}
\sum_{i=0}^{5} A_{i} I^{i}=A_{5}\left(I^{2}+m_{1} I+n_{1}\right)\left(I^{2}+u I+v\right)(I-\alpha) \\
=A_{5}\left(I^{5}+\left(m_{1}+u-\alpha\right) I^{4}+\left(v+n_{1}+m_{1} u-m_{1} \alpha-u \alpha\right) I^{3}\right. \\
\left.+\left(m_{1} v+n_{1} u-n_{1} \alpha-m_{1} u \alpha-v \alpha\right) I^{2}+\left(n_{1} v-\alpha m_{1} v-n_{1} u \alpha\right) I-n_{1} v \alpha\right) .
\end{gathered}
$$

Equating coefficients of like powers on the left and the right we find

$$
\alpha=m_{1}+u-\frac{A_{4}}{A_{5}}=-\left(\frac{A_{4}}{A_{5}}+2 \operatorname{Re}\left(\eta_{1}\right)+2 \operatorname{Re}\left(\omega_{1}\right)\right)=\frac{-A_{0}}{n_{1} v A_{5}}=\frac{-A_{0}}{A_{5}\left|\omega_{1}\right|^{2}\left|\eta_{1}\right|^{2}} .
$$

For feasibility of $I_{5}$, we need $\alpha>0$, i.e. feasibility of the interior equilibrium is given by

$$
\lambda>h_{2}-h_{1}, \quad \frac{\delta^{[4]}}{\delta^{[5]}}<\delta<\frac{\delta^{[3]}}{\delta^{[6]}}
$$


with

$$
\begin{array}{r}
\delta^{[4]}=x_{I V 5}^{2} h_{2} q+x_{I V 5} e m p h_{1}+p m x_{I V 5} e \lambda-x_{I V 5} h_{2} e m+x_{I V 5} h_{2} q b \\
+h_{2} q a^{2}, \\
\delta^{[6]}=\left(a^{2} h_{1}^{2}(1-p)+a^{2} \lambda h_{1}\right)+x_{I V 5} h_{1} b \lambda+x_{I V 5}^{2}\left(h_{1}^{2}+\lambda h_{1}-h_{1}^{2} p\right)+x_{I V 5} h_{1}^{2} b(1-P), \\
\delta^{[3]}=\left(-a^{2} \lambda^{2} q-x_{I V 5}^{2} \lambda^{2} q+x_{I V 5} e m h_{1} h_{2} p+x_{I V 5} h_{1} \lambda p q b\right. \\
-x_{I V 5} h_{1} h_{2} p q b-a^{2} h_{1} h_{2} p q+a^{2} h_{1} \lambda p q-x_{I V 5}^{2} h_{1} h_{2} p q+x_{I V 5}^{2} h_{1} \lambda p q \\
-x_{I V 5} h_{1}^{2} e m p^{2}+x_{I V 5} h_{2} \lambda q b-x_{I V 5} h_{1} h_{2} e m-x_{I V 5} h_{2} \lambda e m \\
+a^{2} h_{1} h_{2} q+x_{I V 5} h_{1} h_{2} q b-x_{I V 5} \lambda h_{1} q b+x_{I V 5} \lambda h_{1} e m+x_{I V 5} h_{1}^{2} e m p-a^{2} \lambda q h_{1} \\
\left.+a^{2} \lambda h_{2} q-x_{I V 5}^{2} h_{1} \lambda q+x_{I V 5}^{2} h_{1} h_{2} q+x_{I V 5}^{2} \lambda h_{2} q+x_{I V 5} \lambda^{2} e m-x_{I V 5} \lambda^{2} q b\right) .
\end{array}
$$

\subsubsection{Proof of Proposition 2}

Proof of Proposition 2. Let $R_{x}^{3}=\left\{(x, y, z) \in R_{x}^{+}, x \geq 0, y \geq 0, z \geq 0\right\}$, consider the scalar function $L_{I}: R_{x}^{3} \rightarrow R$ defined by

$$
L_{I}(t)=k_{1}\left(x-x_{I 5}-x_{I 5} \ln \frac{x}{x_{I 5}}\right)+\frac{1}{2}\left(y-y_{I 5}-y_{I 5} \ln \frac{y}{y_{I 5}}\right)+k_{3}\left(z-z_{I 5}-z_{I 4} \ln \frac{z}{z_{I 5}}\right) .
$$

The derivative of Eq. (4.13) along the solutions of (2.4) is

$$
\frac{d L_{I}}{d t}=\frac{d L_{I 1}(x, y, z)}{d t}+\frac{d L_{I 2}(x, y, z)}{d t}+\frac{d L_{I 3}(x, y, z)}{d t} .
$$

Now

$$
\begin{aligned}
\frac{d L_{I 1}}{d t} & =k_{1}\left(x-x_{I 5}\right)(r-\beta x-m y-p m z) \\
& =k_{1}\left(x-x_{I 5}\right)\left[-\beta\left(x-x_{I 5}\right)-m\left(y-y_{I 5}\right)-p m\left(z-z_{I 5}\right)\right], \\
\frac{d L_{I 2}}{d t} & =\frac{1}{2}\left(y-y_{I 5}\right)\left(e m x-\lambda z-q-h_{1}(y+z)\right) \\
& =\frac{1}{2}\left(x-x_{I 5}\right)\left[e m\left(x-x_{I 5}\right)-\left(\lambda+h_{1}\right)\left(z-z_{I 5}\right)-h_{1}\left(y-y_{I 5}\right)\right], \\
\frac{d L_{I 3}}{d t} & =k_{3}\left(z-z_{I 5}\right)\left[\lambda y+e m p x-\delta-h_{2}(y+z)\right] \\
& =k_{3}\left(z-z_{I 5}\right)\left[e m p\left(x-x_{I 5}\right)+\left(\lambda-h_{2}\right)\left(y-y_{I 5}\right)-h_{2}\left(z-z_{I 5}\right)\right] .
\end{aligned}
$$


Therefore

$$
\begin{aligned}
\frac{d L}{d t}= & -k_{1} \beta\left(x-x_{I 5}\right)^{2}+\left(\frac{e m}{2}-m k_{1}\right)\left(x-x_{I 5}\right)\left(y-y_{I 5}\right)+\left(k_{3} e m p-p m k_{1}\right)\left(x-x_{I 5}\right)\left(z-z_{I 5}\right) \\
+ & {\left[k_{3}\left(\lambda-h_{2}\right)-\frac{\lambda+h_{1}}{2}\right]\left(y-y_{I 5}\right)\left(z-z_{I 5}\right)-\frac{h_{1}}{2}\left(y-y_{I 5}\right)^{2}-h_{2} k_{3}\left(z-z_{I 5}\right)^{2} } \\
\leq & {\left[\frac{k_{3} e m p-p m k_{1}}{2}-k_{1} \beta\right]\left(x-x_{I 5}\right)^{2}+\left[k_{3} \frac{\lambda-h_{2}}{2}-\frac{\lambda+h_{1}}{4}\right.} \\
& \left.-\frac{h_{1}}{2}\right]\left(y-y_{I 5}\right)^{2}+\left[k_{3} \frac{\lambda-h_{2}}{2}-\frac{\lambda+h_{1}}{4}-h_{2} k_{3}\right. \\
& \left.+\frac{k_{3} e m p-p m k_{1}}{2}\right]\left(z-z_{I 5}\right)^{2} \leq 0,
\end{aligned}
$$

having taken

$$
k_{1}=\frac{e}{2}, \quad k_{3}=\frac{\lambda+3 h_{1}}{2\left(\lambda-h_{1}\right)}, \quad k_{3} \leq \frac{\lambda+h_{1}+e m p}{2\left(\lambda-3 h_{2}+e m p\right)}, \quad k_{3} \leq \frac{m p+2 \beta}{2 m p},
$$

which correspond to the assumptions (2.13), (2.14). Also the derivative vanishes only at the equilibrium $E_{5}{ }^{I}$. Thus, $L_{I}$ is a Lyapunov function and by Lasalle theorem global stability follows.

\subsubsection{The $b_{1}$ is given by}

$$
\begin{array}{r}
b_{1}=x_{I V 5}^{2} m \delta\left(\lambda+h_{1}\right)-x_{I V 5}^{2} h_{2} q m-x_{I V 5} e m^{2} p h_{1}+x_{I V 5} \delta b m h_{1}-p m^{2} x_{I V 5} e \lambda \\
+x_{I V 5} h_{2} e m^{2}+m \lambda \delta b x_{I V 5}-x_{I V 5} m h_{2} q b+\delta a^{2} m \lambda+\delta a^{2} m h_{1}-h_{2} q a^{2} m, \\
d_{1}=a^{2} \lambda^{2} q+a^{2} \delta h_{1}^{2}+x_{I V 5}^{2} \lambda^{2} q+x_{I V 5}^{2} \delta h_{1}^{2}-x_{I V 5} e m h_{1} h_{2} p-x_{I V 5} h_{1} \lambda p q b \\
\quad+x_{I V 5} h_{1} h_{2} p q b+a^{2} h_{1} h_{2} p q-a^{2} h_{1} \lambda p q+x_{I V 5}^{2} h_{1} h_{2} p q-x_{I V 5}^{2} h_{1} \lambda p q \\
+x_{I V 5} h_{1}^{2} e m p^{2}+x_{I V 5} h_{1} \delta b \lambda-x_{I V 5} h_{2} \lambda q b-x_{I V 5} h_{1}^{2} \delta b p+x_{I V 5} h_{1} h_{2} e m \\
+x_{I V 5} h_{2} \lambda e m-x_{I V 5} h_{1} h_{2} q b+x_{I V 5} \lambda h_{1} q b-x_{I V 5} \lambda h_{1} e m-x_{I V 5} h_{1}^{2} e m p \\
+a^{2} \lambda \delta h_{1}+a^{2} \lambda q h_{1}-a^{2} h_{1} h_{2} q-a^{2} h_{1}^{2} \delta p-a^{2} \lambda h_{2} q+x_{I V 5}^{2} \delta \lambda h_{1}+x_{I V 5}^{2} h_{1} \lambda q \\
-x_{I V 5}^{2} h_{1} h_{2} q-x_{I V 5}^{2} h_{1}^{2} \delta p-x_{I V 5}^{2} \lambda h_{2} q-x_{I V 5} \lambda^{2} e m+x_{I V 5} \lambda^{2} q b+x_{I V 5} h_{1}^{2} \delta b
\end{array}
$$

